Corrected: Author Correction

ARTICLE

https://doi.org/10.1038/s41467-018-08182-x

\title{
Loss-of-function mutations in QRICH2 cause male infertility with multiple morphological abnormalities of the sperm flagella
}

\author{
Ying Shen', Feng Zhang (10) 2,3,4 Fuping Li ${ }^{5,6}$, Xiaohui Jiang 5,6, Yihong Yang ${ }^{7}$, Xiaoliang Li', Weiyu Li², \\ Xiang Wang ${ }^{1}$, Juan Cheng ${ }^{8}$, Mohan Liu ${ }^{9}$, Xueguang Zhang ${ }^{1}$, Guiping Yuan ${ }^{10}$, Xue Pei ${ }^{9}$, Kailai Cai ${ }^{11}$, Fengyun Hu ${ }^{12}$, \\ Jianfeng Sun ${ }^{13}$, Lanzhen Yan ${ }^{14}$, Li Tang ${ }^{1}$, Chuan Jiang ${ }^{1}$, Wenling Tu ${ }^{9}$, Jinyan $\mathrm{Xu}^{5,6}$, Haojuan $\mathrm{Wu}^{8}$, Weiqi Kong ${ }^{1}$, \\ Shuying $\mathrm{Li}^{1}$, Ke Wang${ }^{1}$, Kai Sheng ${ }^{1}$, Xudong Zhao ${ }^{14,15}$, Huanxun Yue ${ }^{5,6}$, Xiaoyu Yang ${ }^{16}$ \& Wenming Xu ${ }^{1}$
}

\begin{abstract}
Aberrant sperm flagella impair sperm motility and cause male infertility, yet the genes which have been identified in multiple morphological abnormalities of the flagella (MMAF) can only explain the pathogenic mechanisms of MMAF in a small number of cases. Here, we identify and functionally characterize homozygous loss-of-function mutations of $\mathrm{QRICH} 2$ in two infertile males with MMAF from two consanguineous families. Remarkably, Qrich2 knock-out (KO) male mice constructed by CRISPR-Cas9 technology present MMAF phenotypes and sterility. To elucidate the mechanisms of Qrich2 functioning in sperm flagellar formation, we perform proteomic analysis on the testes of $\mathrm{KO}$ and wild-type mice. Furthermore, in vitro experiments indicate that QRICH2 is involved in sperm flagellar development through stabilizing and enhancing the expression of proteins related to flagellar development. Our findings strongly suggest that the genetic mutations of human QRICH2 can lead to male infertility with MMAF and that QRICH2 is essential for sperm flagellar formation.
\end{abstract}

\footnotetext{
${ }^{1}$ Department of Obstetrics/Gynecology, Joint Laboratory of Reproductive Medicine (SCU-CUHK), Key Laboratory of Obstetric, Gynecologic and Pediatric Diseases and Birth Defects of Ministry of Education, West China Second University Hospital, Sichuan University, 610041 Chengdu, China. ${ }^{2}$ Obstetrics and Gynecology Hospital, Institute of Metabolism and Integrative Biology, School of Life Sciences, Fudan University, 200011 Shanghai, China. ${ }^{3}$ State Key Laboratory of Reproductive Medicine, Center for Global Health, School of Public Health, Nanjing Medical University, 211116 Nanjing, China. ${ }^{4}$ Shanghai Key Laboratory of Female Reproductive Endocrine Related Diseases, 200011 Shanghai, China. ${ }^{5}$ Human Sperm Bank, West China Second University Hospital, Sichuan University, 610041 Chengdu, China. ${ }^{6}$ Key Laboratory of Birth Defects and Related Disease of Women and Children (Sichuan University), Ministry of Education, 610041 Chengdu, China. ${ }^{7}$ Center of Reproductive Medicine, West China Second University Hospital, Sichuan University, 610041 Chengdu, China. ${ }^{8}$ Southwest Medical University, 646000 Luzhou, China. ${ }^{9}$ Department of Medical Genetics, State Key Laboratory of Biotherapy, West China Hospital, Sichuan University and Collaborative Innovation Center, 610041 Chengdu, China. ${ }^{10}$ Analytical \& Testing Center, Sichuan University, 610041 Chengdu, China. ${ }^{11}$ Chengdu Research Base of Giant Panda Breeding, 610041 Chengdu, China. ${ }^{12}$ Auckland University, 1142 Auckland, New Zealand. ${ }^{13}$ Teaching Hospital of Chengdu University of TCM, 610041 Chengdu, China. ${ }^{14}$ Key Laboratory of Animal Models and Human Disease Mechanisms of Chinese Academy of Sciences and Yunnan Province, Kunming Institute of Zoology, Chinese Academy of Sciences, 650000 Kunming, China. ${ }^{15}$ Center for Excellence in Animal Evolution and Genetics, Chinese Academy of Sciences, 650223 Kunming, China. ${ }^{16}$ State Key Laboratory of Reproductive Medicine, Clinical Center of Reproductive Medicine, The First Hospital, Nanjing Medical University, 210029 Nanjing, China. Correspondence and requests for materials should be addressed to X.Z. (email: zhaoxudong@mail.kiz.ac.cn) or to H.Y. (email: yuehuanx@139.com) or to X.Y. (email: yxy1921@163.com) or to W.X. (email: xuwenming@scu.edu.cn)
} 
S perm defects are the direct causes of male infertility, which includes decreased sperm count, reduced sperm motility and abnormal morphology $y^{1,2}$. Sperm motility is a critical factor for normal fertilization, and over $80 \%$ of male infertility is caused by impaired sperm motility ${ }^{3}$. Sperm morphology plays an essential role in sperm locomotion, and defective sperm morphology makes an assignable contribution to male infertility, which consists of a wide range of phenotypes involving the head, neck, mid-piece or tail of the spermatozoa. Among these, multiple morphological abnormalities of the sperm flagella (MMAF) is characterized by a mosaic of morphological abnormalities of the flagellum including coiled, bent, irregular, short or/and absent flagella ${ }^{4-6}$. Normal sperm flagella contain a $9+2$ axonemal arrangement of nine peripheral microtubule doublets (OD) and two central microtubules (CP), surrounded by outer dense fiber (ODF) and fibrous sheath (FS $)^{7}$. Almost all MMAF cases are accompanied by visible ultrastructural derangement and/or defects, such as incomplete or disorganized axoneme arrangement or absence of ODF/FS $5,6,8$. Any defects in these structures would result in aberrant sperm morphology and further lead to reduced motility or even sperm immobility.

Research is ongoing to investigate the molecular mechanisms of MMAF. Using knock-out (KO) mouse models, several genes have been found to be associated with sperm flagellar development in mice ${ }^{9}$. The complete ablation of Spata6 caused impaired development of the connecting piece and tight head-tail conjunction ${ }^{10}$. Subtle disorganization of the ultrastructure was observed in Tekt4-null mice ${ }^{11}$. The sperm flagella of Tssk4-absent mice represented morphological defects at the midpiece-principal piece junction $^{12}$. Mice lacking Odf2 showed axoneme defects ${ }^{13}$. Ropn1-deficient mice exhibited structural defects in the principal piece of sperm flagellum ${ }^{14}$. A significant disorganization in the fibrous sheath, as well as abnormal configuration of doublet microtubules, was observed in Cabyr-knockout male mice ${ }^{15}$. Although MMAF is not a rare autosomal-recessive inherited disorder in humans, the genetic etiology of a large proportion of MMAF cases remains elusive ${ }^{4-6}$. To date, mutations in only six genes have been determined in humans related to MMAF: AKAP4, CCDC39, DNAH1, CFAP43, CFAP44, and CFAP69 (refs. ${ }^{4,5,8,16-19}$ ); however, these could explain the mechanism of only a few MMAF cases. Therefore, it remains necessary to improve the discriminative power of a single candidate gene in human MMAF.

In this study, two homozygous nonsense mutations of the glutamine rich 2 (QRICH2) gene are identified in two MMAF patients from two consanguineous families through whole-exome sequencing (WES). The Qrich2 KO mouse model represents typical MMAF phenotypes including coiled, bent, irregular, short or/and absent flagella and defects of sperm flagellar ultrastructure. These phenotypes are consistent with those observed in human subjects who carried the loss-of-function mutations of QRICH2. This study provides compelling evidence that the loss-of-function mutations of QRICH2 could result in MMAF and cause male infertility, and QRICH2 is a functional molecule essential for sperm flagellar development by regulating the genes associated with the accessory structure of sperm flagella.

\section{Results}

Loss-of-function mutations of QRICH2 in males with MMAF. Two infertile males from two consanguineous families were investigated in our study (Fig. 1a, b). Semen analysis is presented in Table 1 . The sperm quantity was basically normal, whereas abnormalities in the spermatozoa tail region were up to $99 \%$. Therefore, almost no progressive motility of spermatozoa was observed in these two cases. Scanning electron microscopy (SEM) further identified the MMAF phenotypes of these two cases. We observed some spermatozoa with short tails, some with thick short tails, and some with coiled tails, and some spermatozoa appeared to have only the head region (Fig. 1c). In addition, a variety of ultrastructural defects were detected in the sperm flagella under transmission electron microscopy (TEM) (Fig. 1d). For patient A (PA) integrated and regularly arranged OD and ODF were observed, whereas the CP were missed in the midpiece of most flagella. In the principal piece of most flagella, some ODF and OD were absent, the remainders were disorganized, and the CP could not be observed as well. For patient B (PB) the atypical $6+0$ composition of axonemal microtubules was shown in the flagellar mid-piece; additionally, a complete lack of CP and the irregular arrangement of the OD and ODF were revealed in the principal piece.

The previous studies have reported that the homozygous lossof-function mutations of several genes could cause MMAF and have suggested that MMAF is a disorder with an autosomalrecessive inheritance pattern ${ }^{5,8,16,19}$. According to this, we performed WES on the two males to elucidate the underlying genetic causes of the MMAF phenotypes, and many homozygous variants were found in the two patients (Supplementary Data 1). Briefly, variants were removed if (1) the minor allele frequency was $\geq 1 \%$ in any database, including the gnomAD, ExAC Browser and 1000 Genomes Project, because the pathogenic variants responsible for MMAF are rare in human populations; (2) the heterozygous variant, because MMAF has been assumed to be an autosomal-recessive inheritance; (3) the variant affected: $3^{\prime}$ or $5^{\prime}$ untranslated regions, noncoding exons, or intronic sequences except canonical splice sites; and (4) the variant was not predicted damaging by PolyPhen-2, SIFT and MutationTaster tools. Subsequently, the selected genes harboring candidate pathogenic variants from WES were evaluated by Human Protein Atlas (http://www.proteinatlas.org), which contains tissue-specific protein expression data, and the genes which are primarily or specifically expressed in the testis were included in our research. Remarkably, after exclusion of frequent variants and application of technical and biological filters, two homozygous nonsense mutations were identified in QRICH2 (Table 1): a homozygous stop-gain mutation in exon 3 (c.192G $>A$ [p. L64 ${ }^{*}$ ) in the individual $\mathrm{PA}$ and a homozygous stop-gain variant in exon 4 in the individual PB (c.3037C>T [p.R1013*]). To clarify the putative contribution of these mutations to the MMAF phenotypes, we investigated the $\mathrm{QRICH} 2$ gene by Sanger sequencing in PA and another affected individual (IV:3 and IV:5), their healthy parents (III:3 and III:4), and unaffected siblings (IV:2, IV:8 and IV:9) from Family A. In this family, the another affected individual (IV:5) carried the homozygous mutation (c.192G>A) and their healthy parents (III:3 and III:4), as well as one unaffected sibling (IV:8) carried the heterozygous mutation (c.192G $>$ A) (Supplementary Fig. 1a). For family $B$, we detected the heterozygous mutation (c.3037C>T) in their healthy parents (III:1 and III:2) (Supplementary Fig. 1b). The results of DNA sequencing suggested that the two homozygous nonsense mutations of PA and $\mathrm{PB}$ were inherited from their parents carrying heterozygous mutations, which is in line with the nature of MMAF being an autosomal recessive inheritance.

The expression of QRICH2 in sperm flagella. The Human Proteins Atlas Database indicates that the expression of human QRICH2 is restricted to the testes, while no information was available with regard to QRICH2 protein distribution in spermatozoa. To determine the location of QRICH2 on human spermatozoa accurately, we used stochastic optical reconstruction microscopy (STORM), which enables $3 \mathrm{D}$ reconstruction at 
a

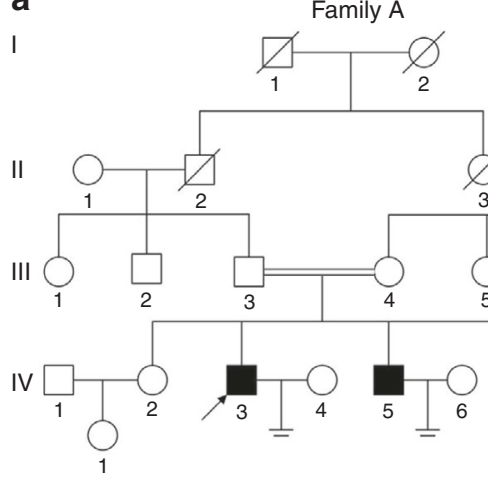

\section{Family A}

C

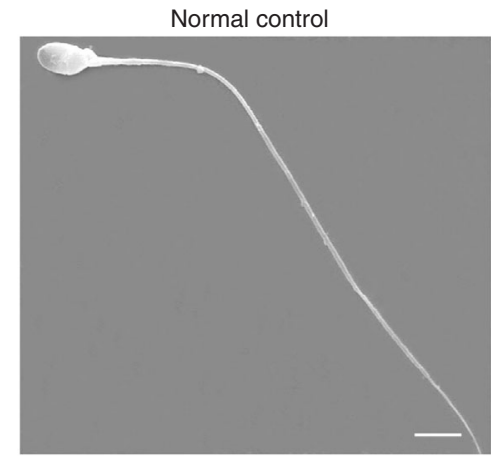

b

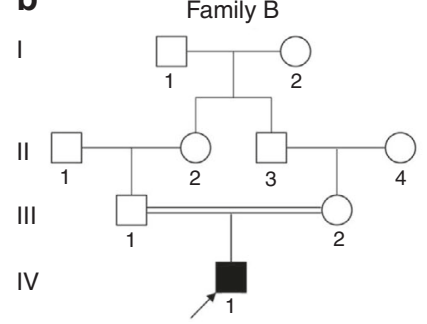

d
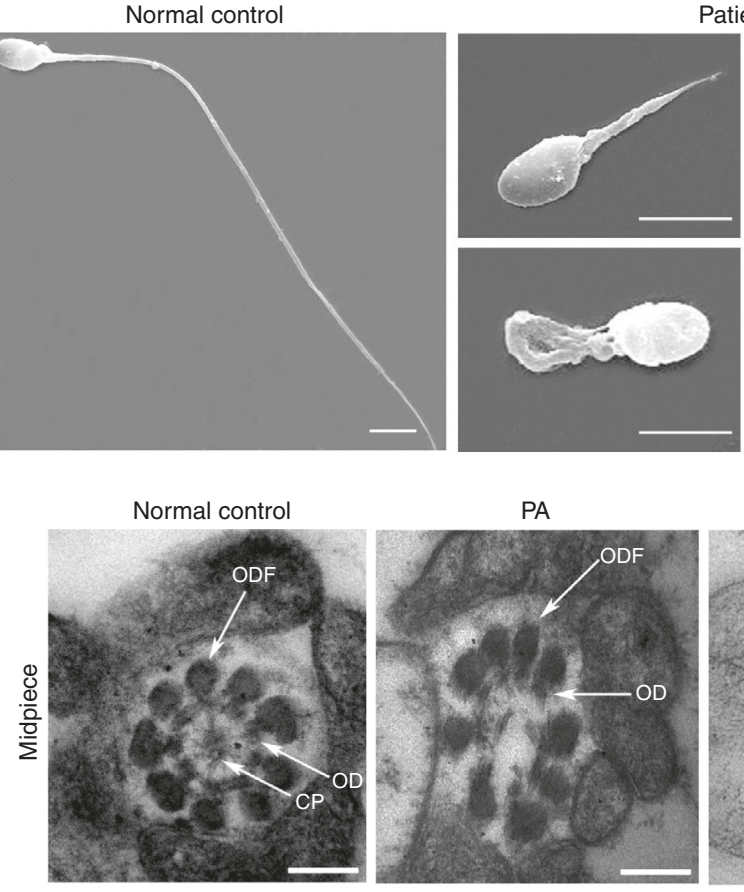

Patients
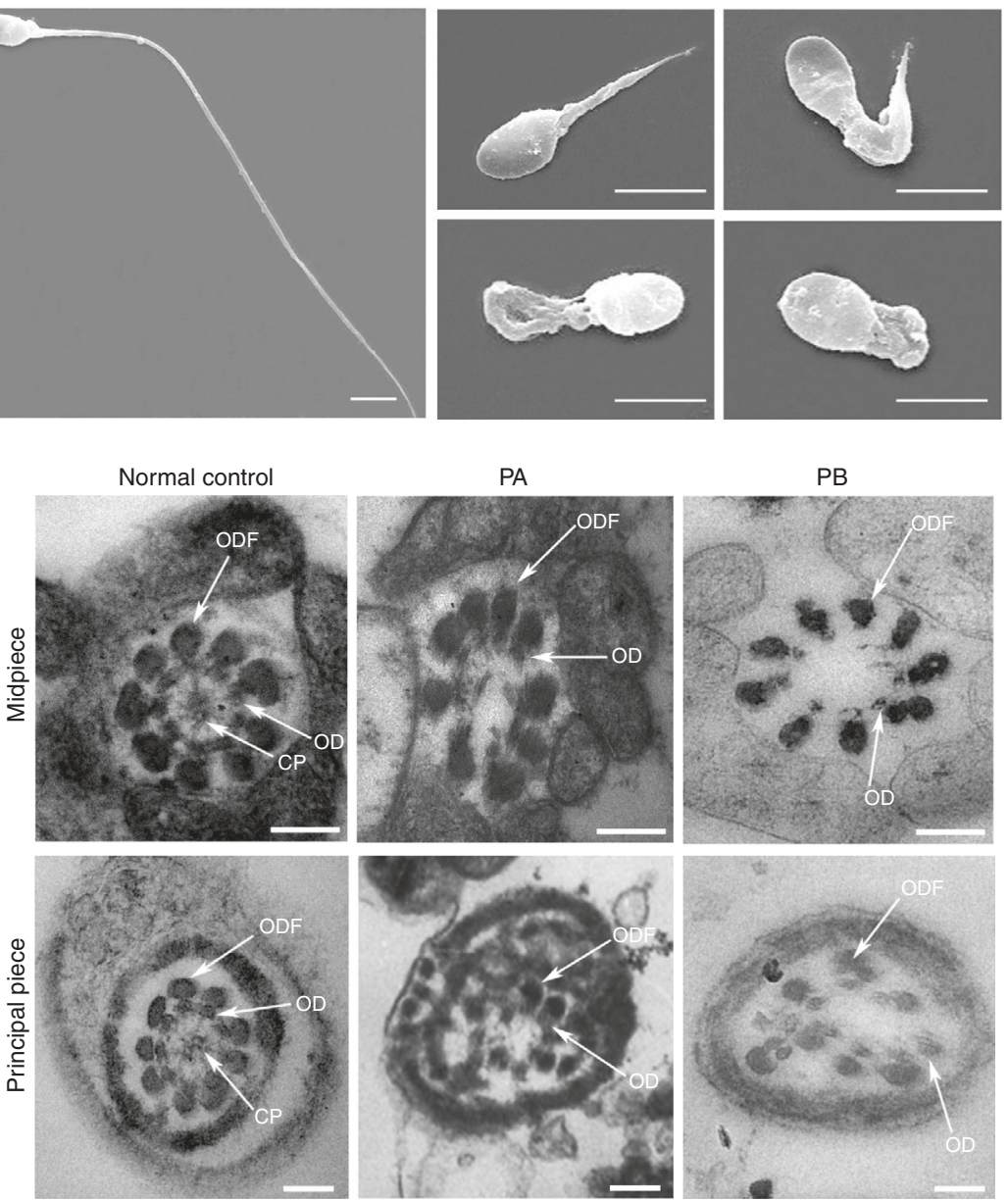

Fig. 1 Sperm morphology and ultrastructure in the two MMAF patients. a, b The pedigree structure and segregation analysis in two families. Squares represent male pedigree members, circles represent female pedigree members, solid symbols represent members with MMAF, and open symbols represent unaffected members; the probands are indicated by black arrows. c The malformations of sperm flagella in the two MMAF individuals. The absent, short, thick, or coiled flagella and other MMAF phenotypes were observed in two patients compared with the normal control by SEM (scale bars, $5 \mu \mathrm{m})$. d The abnormal ultrastructures of the mid-piece and principal piece of sperm flagella in two patients. By TEM, in the mid-piece, PA exhibited an atypical $9+0$ arrangement of axonemal microtubules, and the CP and OD defects were observed in PB. The principal piece of the PA and PB flagella consist of incomplete and disorganized OD and ODF and lack CP. Fibrous sheaths were not apparent, especially for PB. (scale bars, $100 \mathrm{~nm}$ )

$10-20 \mathrm{~nm}$ or higher resolutions ${ }^{20-23}$. As expected, 3D STORM images of QRICH2 showed a continuous distribution along the sperm flagella (Fig. 2a-c, Supplementary Movie 1). Notably, QRICH2 co-localized with microtubule protein $\alpha$-Tubulin which is an axonemal component, indicating that QRICH2 is expressed in sperm tail and plays a vital role in sperm flagellar development (Fig. 2d-i, Supplementary Movies 2, 3).
The QRICH2 gene is localized on human chromosome 17, contains 19 exons and encodes a predicted 1663-amino acid protein (NP_115510.1) with three principal domains (Fig. 2j). The homozygous stop-gain mutation of c.192G>A [p. L64 ${ }^{\star}$ ] is located in exon 3 of $Q R I C H 2$ which causes premature translational termination of QRICH2 protein resulting in the absence of three domains of QRICH2 (Fig. 2d). The other homozygous 


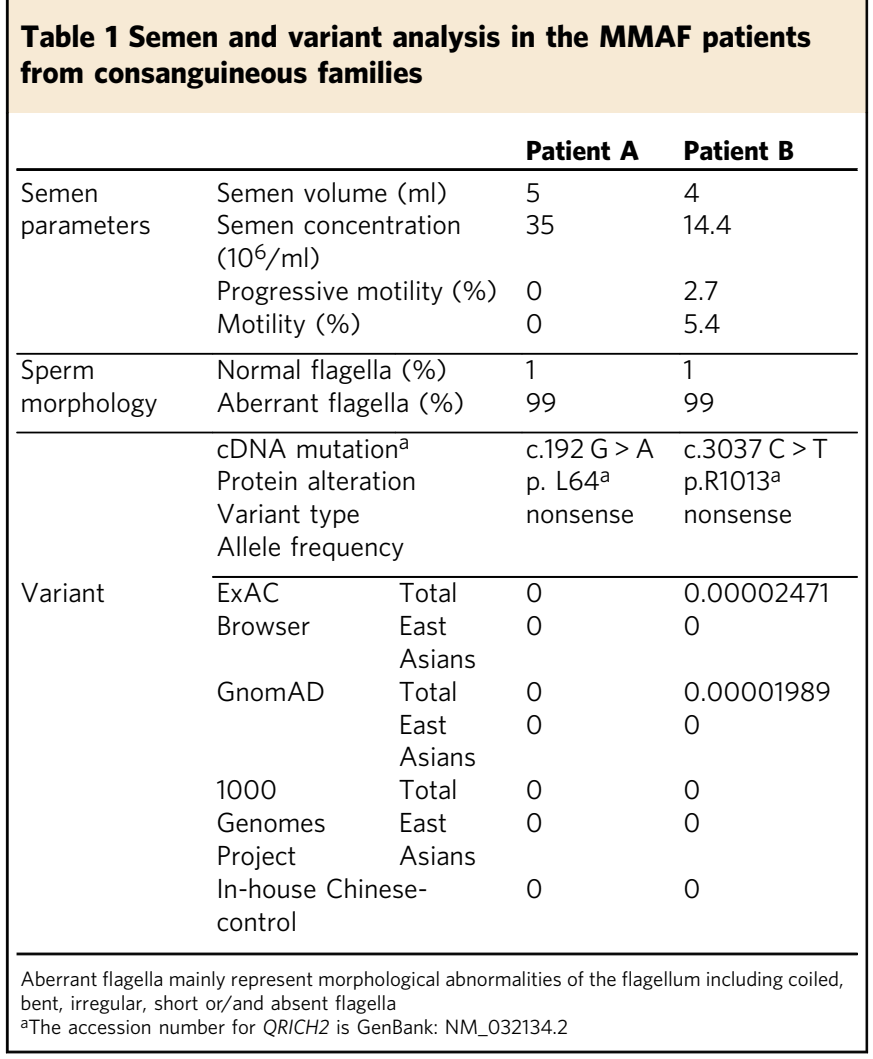

stop-gain variant, c.3037C > T [p.R1013*], which is located in exon 4 of QRICH2, results in the loss of SMC_N super family domain and DUF4795 domain (Fig. 2j). To confirm the effect of the two mutations on QRICH2 expression, we performed immunofluorescence assays on spermatozoa of the two MMAF patients. The QRICH2 protein was not detected in spermatozoa from PA and was remarkably weak in spermatozoa from PB (Fig. 2k). Thus, the two homozygous stop-gain variants did impact the expression of QRICH2 negatively, leading to the abnormal development of sperm flagella.

Lacking Qrich2 leads to MMAF in male mice. QRICH2 is highly conserved during evolution. The human QRICH2 shares 96.7, $89.5,74.2,61.2,81.8$, and $59.5 \%$ of protein identity with its orthologs in chimpanzees, rhesus monkeys, dogs, cattle, mice and rats, respectively (https://www.ncbi.nlm.nih.gov) (Supplementary Fig. 2a, b). The expression profile of Qrich2 in adult mice suggested that Qrich2 is primarily expressed in the testis tissue (Supplementary Fig. 2c). Accurately, immunofluorescent stain of testis sections from WT mice showed that Qrich2 is mainly expressed in the round and elongated spermatids during the spermiogenesis, and the Qrich2 staining is absent in the testis sections from KO mice (Supplementary Fig. 3a-d). To better understand the localization of Qrich2 in different steps of germ cells, we carried out flow cytometry coupled to cell sorting assay to isolate elongated spermatids, round spermatids and spermatogonia. The immunofluoresence results indicated that Qrich2 is distributed in the nuclear membrane of the spermatogonia (Supplementary Fig. 3e), in the nucleus of the round spermatids (Supplementary Fig. 3f), in the nucleus and cytoplasm of the early elongating spermatids (Supplementary Fig. 3g), in the cytoplasm of late elongating spermatids (Supplementary Fig. 3h) and in the flagella of epididymal spermatozoa (Supplementary Fig. 3i). Furthermore, to find out the temporal expression of Qrich2, Qrich2 expression levels were investigated in mice testes at
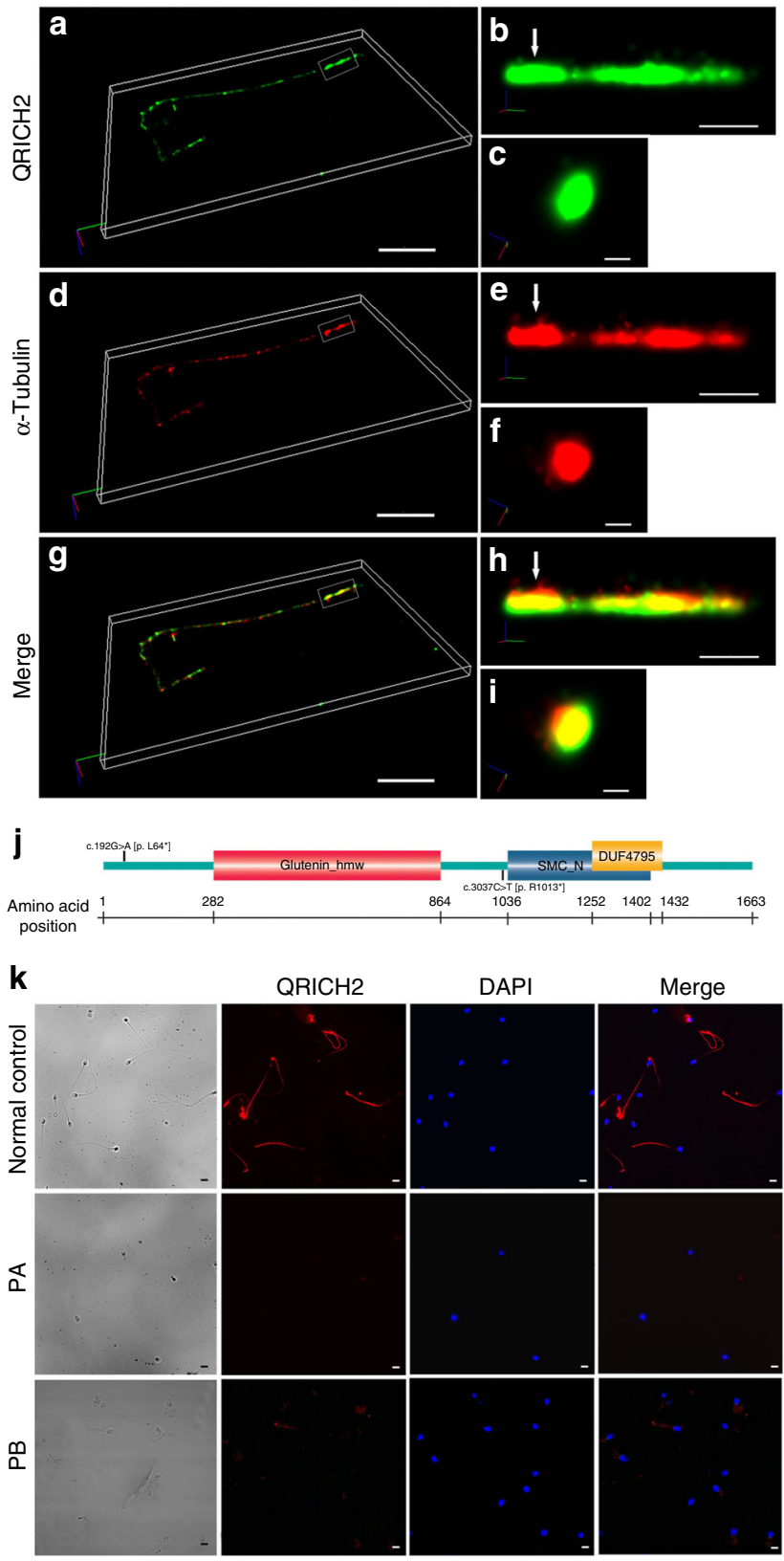

Fig. 2 QRICH2 is detected in human sperm flagella. a-i 3D STORM images of QRICH2 (green) (a-c), $\alpha$-Tubulin (red) (d-f) and their co-localization (yellow) (g-i). a, d, $\mathbf{g}, x-y-z$ projections (scale bars, $5 \mu \mathrm{m}$ ). b, e, $\mathbf{h}, x-y$ projections: sections surrounded by white square (scale bars, $0.5 \mu \mathrm{m}$ ). c, $\mathbf{f}, \mathbf{i}, y-z$ cross-sections near the annulus (white arrowheads) (scale bars, $0.1 \mu \mathrm{m}$ ). $\mathbf{j}$ The domains of QRICH2 and the positions of two mutations in QRICH2. QRICH2 contains three domains: Glutenin hmw super family (amino acid positions 282-864), SMC_N super family (amino acid positions 1036-1402) and DUF4795 (amino acid positions 1252-1432). k Immunofluorescence staining of QRICH2 on human spermatozoa from normal control and the two MMAF individuals. QRICH2 staining (red) was absent in sperm from PA and significantly weak in sperm from PB. (blue, DAPI; scale bars, $5 \mu \mathrm{m}$ )

different postnatal days. The real-time PCR results showed that Qrich2 began to be expressed obviously at postnatal day 15, reached the peak of expression at postnatal day 22, and then maintained a stable expression level afterward (Supplementary Fig. 2d). Thus, Qrich2 is stably, highly and exclusively expressed in adult mice testes during evolution. 
Considering that Qrich2 protein in mice is highly homologous to human QRICH2 protein and primarily expressed in mice testes, we established Qrich2-KO mice using the CRISPR-Cas9 technology to define the physiological role of QRICH2 in sperm flagellar formation. A frameshift mutation was generated by deleting exon 1 and exon 2 of Qrich 2 to achieve the knock out (Fig. 3a). We performed PCR, real-time PCR and western blotting to confirm the success of the Qrich2-KO mice (Supplementary

a

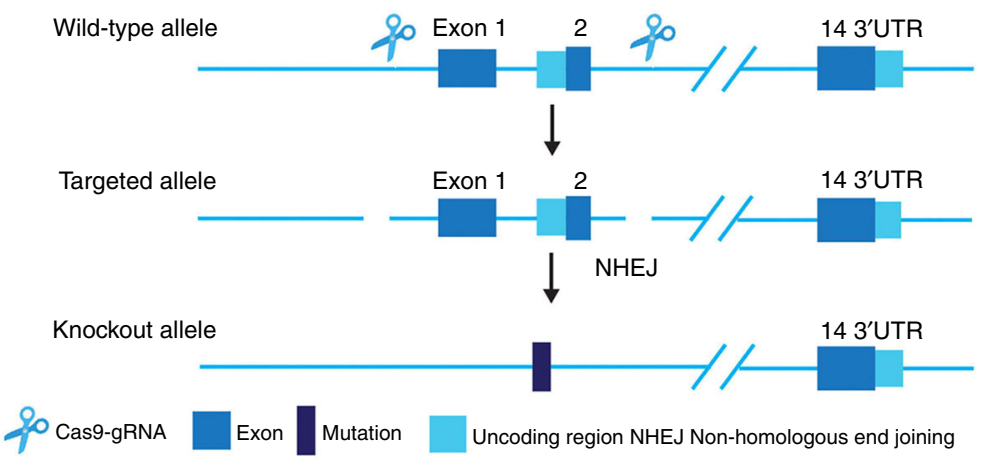

b

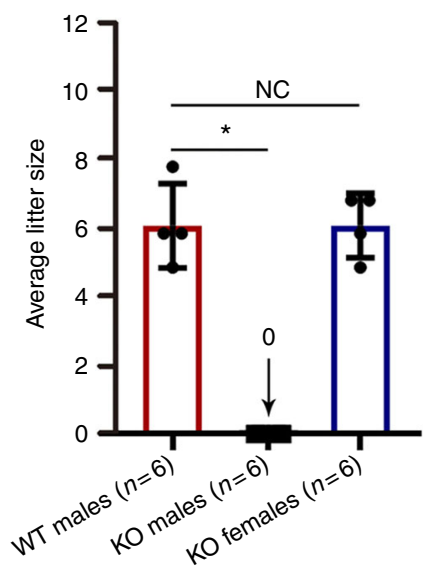

WT

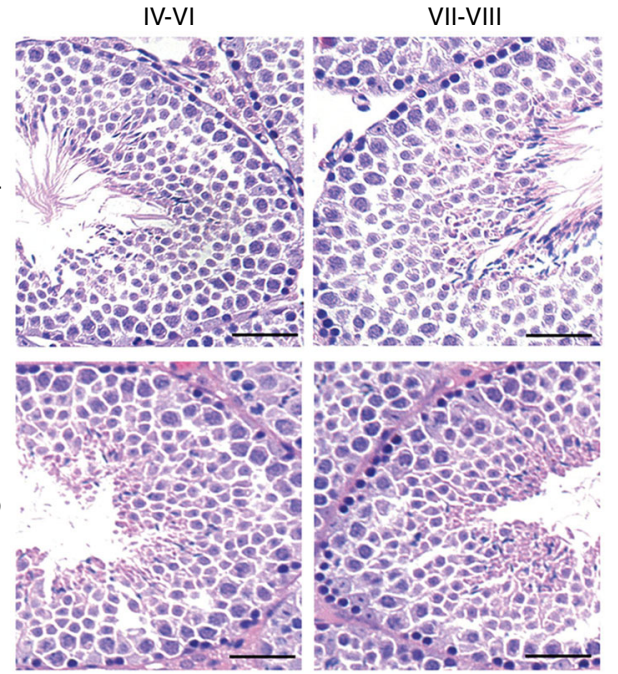

d

Cauda epididymidis
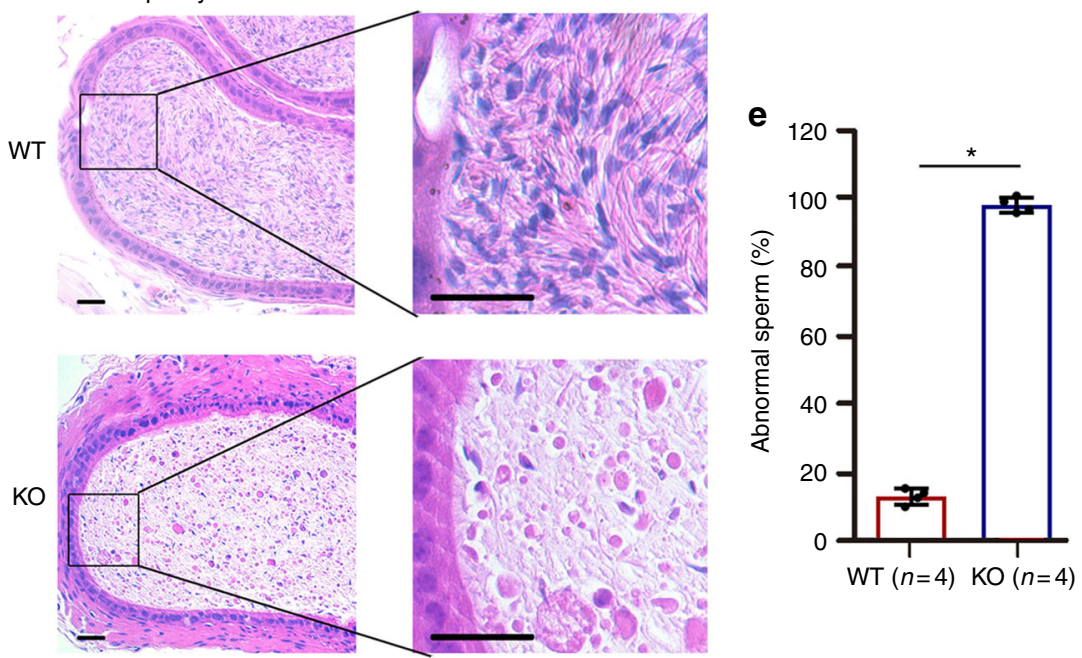

Fig. 3 Deficiency of Qrich2 leads to MMAF in mice. a The schematic illustration of the targeting strategy for generating Qrich2-knockout mice. A frameshift mutation was generated by deleting exon 1 and exon 2 of Qrich2. b Fertility of wild-type (WT), knock out (KO) males and knock out (KO) females. KO male mice and KO female mice were bred with the WT mice and the average litter size was measured. KO males were completely sterile. (Student's $t$-test; ${ }^{\star} p<0.05$; NS, not significant; error bars, s.e.m) c The development of sperm flagella in stages IV-VIII of spermatogenesis in mice testes by H\&E staining (scale bars, $20 \mu \mathrm{m}$ ). Lack of elongated flagella was observed in the spermatozoa of the KO mice compared with the WT mice. $\mathbf{d}$ The dysplasia of the sperm flagella and decreased sperm quantity were observed in the cauda epididymidis of the KO mice (scale bars, $10 \mu \mathrm{m}$ ). e The number of abnormal sperm in WT males and KO males. Almost $100 \%$ of sperm in KO males displayed abnormal flagella. (Student's $t$-test; ${ }^{\star} p<0.05$; error bars, s.e.m) 
Fig. 4a-c). After determining the deficiency of Qrich2 in the KO mice, we first investigated the fertility of the $\mathrm{KO}$ mice. The $\mathrm{KO}$ male mice and KO female mice were bred with the WT mice for 5 months. After the mating period, we observed that the KO male mice were infertile, whereas the $\mathrm{KO}$ females had normal fertility (Fig. 3b).

Although no significant differences in testis size and weight were observed between the WT and KO mice, differences in epididymis size and weight were obvious (Supplementary Fig. 4d, e). To determine which spermatogenesis stage that Qrich2 affects on, we compared the histology of testicular tissue sections between the $\mathrm{KO}$ and WT mice by hematoxylin-eosin (H\&E) staining. Flagellar formation defects were observed at stages VIVIII in the KO mice compared with WT mice (Fig. 3c), which indicates that Qrich2 is involved at the stage of sperm flagellar formation. On analyzing the epididymis sections, few spermatozoa were observed, the flagella of which were aberrant in the KO mice (Fig. 3d). Light microscopy first demonstrated the morphological abnormalities of most sperm flagella in the $\mathrm{KO}$ mice (Fig. 3e, Supplementary Fig. 4f). The detailed and clear MMAF phenotypes in the KO mice were revealed by SEM (Fig. 4a). The sperm flagella of $\mathrm{KO}$ mice presented with short, coiled, bent, or other irregular shapes, which were consistent with the clinical phenotypes in the two subjects with MMAF. The ultrastructures of the sperm flagella were analyzed by TEM (Fig. 4b): in the mid-piece of most flagella of the KO mice, displacement of the CP and ODF and absence and/or incorrect localization of the OD were observed; the principal piece in the numerous sperm tails demonstrated missing of the ODF and abnormal $9+2$ structure, for example, the $6+2$ structure. All the defects of sperm flagella observed in the Qrich2-deficient mice strongly support the vital role of QRICH2 in the development of sperm flagella.

In addition, the computer-assisted sperm analysis (CASA) was used to exhaustively examine the sperm quality of the KO mice. The CASA results revealed reduced sperm motility, abnormal sperm flagellar morphology and decreased sperm count in the KO mice compared to the WT mice. Additional parameters showed a significant reduction in sperm locomotion in the $\mathrm{KO}$ mice. The detailed results are presented in Table 2. The diminished sperm movement in the KO mice compared to WT mice is directly reflected in Supplementary Movies 4 and 5. These data together indicate that QRICH2 is involved in sperm flagellar formation and that its deficiency can result in MMAF and further cause male infertility.

QRICH2 regulates flagellum-development-related proteins. To determine the molecular mechanism of QRICH2 during sperm flagellar formation, the protein expression levels in the testes of the WT and KO mice were investigated by a proteomics approach. A total of 6849 proteins were quantified, of which 108 proteins were identified to be expressed differentially, including 92 down-regulated proteins and 19 up-regulated proteins (Fig. 5a). Among the decreased proteins, 33 are involved in the development of sperm flagella, and 15 are required for energy metabolism, which is crucial for sperm motility ${ }^{24-26}$ (Fig. 5b). According to the literature, six of these proteins, namely, A-kinase anchoring protein (AKAP3), outer dense fiber of sperm tails 2 (ODF2), testis specific serine kinase 4 (TSSK4), calcium binding tyrosine phosphorylation regulated (CABYR), rhophilin associated tail protein 1 (ROPN1) and membrane associated ring$\mathrm{CH}$-type finger 10 (MARCH10), were directly related to the formation of the sperm tail; the complete ablation of these proteins caused impaired development of sperm flagella in mouse models ${ }^{10-15}$. Therefore, we focus on these six proteins. Western blotting results further confirmed the decreased expression of these six proteins in the KO mice compared to the WT mice, which was consistent with TMT quantification results (Fig. 5c, d). Meanwhile, the bindings between QRICH2 and AKAP3, ODF2 or TSSK4 were confirmed by exogenous Co-IP in HEK 293 cells (Supplementary Fig. 5a). Furthermore, these proteins, similar to QRICH2, were predominantly localized in the human spermatozoa flagella, supporting their vital roles in sperm tail formation (Fig. 5f).

To confirm the decreased expression of Akap3, Tssk4, Ropn1, March10, Cabyr, and Odf2 was caused by the deficiency of Qrich2 directly, but not by the sperm tail defects, we performed further research on heterozygous (Ht) QRICH2 mice which have the normal flagella development (Supplementary Fig. 5b). The western blotting results showed that the expression of these six proteins in Ht mice is lower than in WT mice (Supplementary Fig. 5c). In addition, when we knocked down QRICH2 in NT2 cells (human testicular embryonic carcinoma cell) which have been identified to express QRICH2, AKAP3, CABYR, ODF2, and TSSK4, and the decreased protein amount of AKAP3, CABYR, ODF2, and TSSK4 was observed in these knocked-down cells compared to the control cells (Supplementary Fig. 5d), Therefore, the decreased levels of the proteins which we focus on are caused by the reduced expression of QRICH2 directly but not the lack of sperm tail structures. Taken together, QRICH2 is involved in sperm tail formation through regulating the expression of sperm tail structural proteins and energy metabolism proteins.

Real-time PCR was used to investigate whether Qrich2 regulated these proteins via their transcriptional levels. We observed that the mRNA levels of Odf2 and Cabyr from KO-mice testes were approximately 60 and 52\% lower than that of WT mice, respectively (Fig. 6a). Meanwhile, the significant decreased mRNA levels of Cabyr/CABRY and Odf2/ODF2 were also observed in Ht mice or QRICH2-knocked-down NT2 cells, compared with WT mice and control cells, respectively (Supplementary Fig. 5e,f). To find out whether QRICH2 acted as a trans-acting factor to enhance the transcriptional activity of $O D F 2$ and $C A B Y R$, chromatin immunoprecipitation (ChIP)-PCR was performed and the results showed that QRICH2 could bind to the promoter regions of $C A B Y R$ and ODF2 (Fig. 6b). Similarly, the ChIP-qPCR results showed the enhanced binding of QRICH2 to the promoter fragments of $O D F 2$ and $C A B Y R$ approximately 4-fold, 11-fold, and 5-fold respectively, compared with the negative control (Fig. 6c, Supplementary Fig. 5g). Furthermore, we constructed luciferase reporter vectors containing $O D F 2$ and $C A B Y R$ promoter fragments and co-transfected them with QRICH2 expression vector individually. The luciferase activity of the constructs with the promoter fragments of ODF2 and $C A B Y R$ were increased in gradient when QRICH2 was overexpressed in gradient in HEK 293 cells (Fig. 6d). These results indicate that QRICH2 enhances ODF2 and CABYR gene expression by acting as a trans-acting factor.

The limited difference in the mRNA levels of Akap3, Tssk4, Ropn1, and March10 between the KO and WT mice indicates that Qrich2 may not affect their transcription (Fig. 6a). Accordingly, we hypothesized that QRICH2 may be involved in the posttranscriptional regulation of these four proteins. The degradation levels of these proteins were determined by analyzing the ubiquitination levels of Akap3, Tssk4, Ropn1, and March10 between the $\mathrm{KO}$ and WT mice, which is the most common mechanism for protein degradation. As expected, the increased ubiquitination degradation of Akap3, Tssk4, Ropn1, and March10 was observed in the KO mice compared with the WT mice (Fig. 6e). We further confirmed the influence of QRICH2 on AKAP3 and TSSK4 by inhibiting their ubiquitination degradation in vitro. We observed that the overexpression of 
a
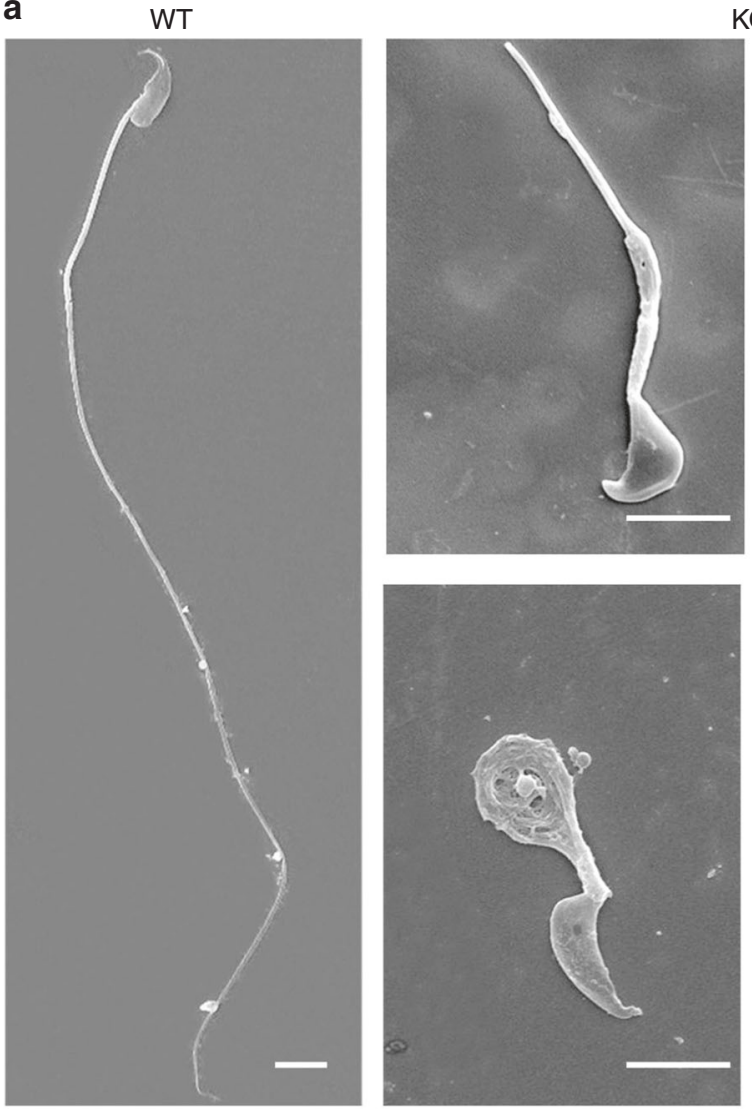

$\mathrm{KO}$
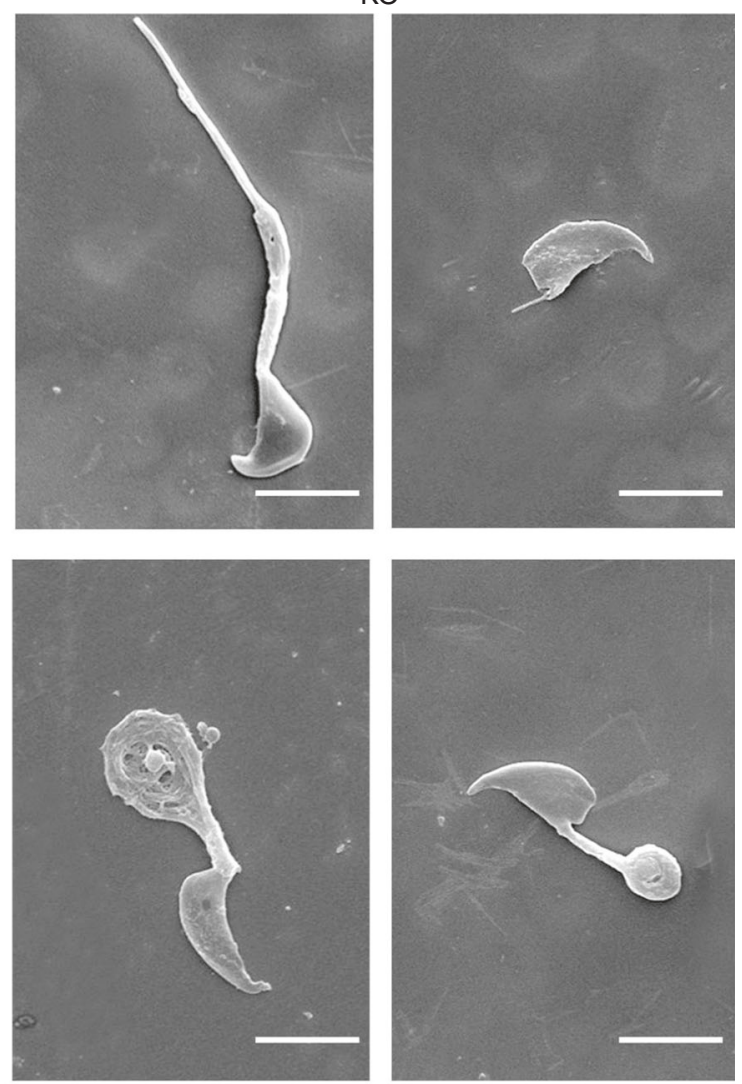

b

WT
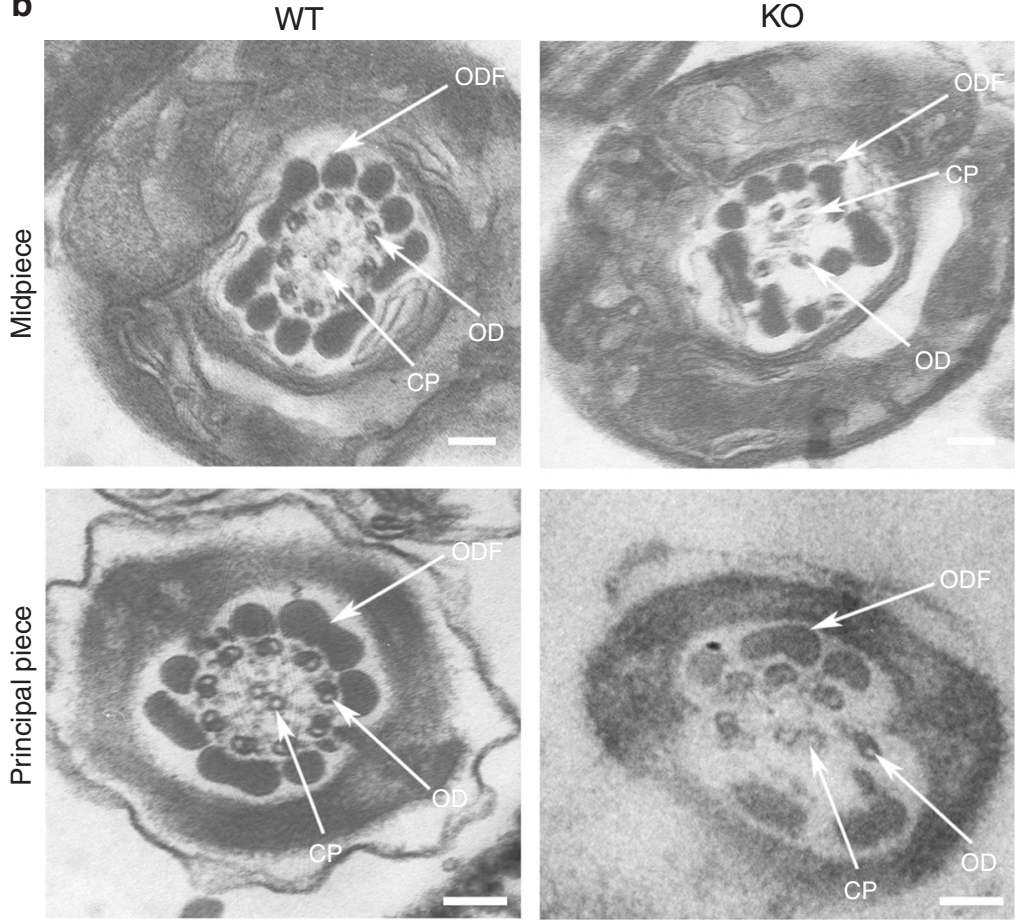

Fig. 4 Electron microscopy analysis shows the typical MMAF phenotypes in KO mice. a The MMAF phenotypes in Qrich2-deficient mice by SEM (scale bars, $5 \mu \mathrm{m}$ ). The WT mice showed a normal flagellum, whereas the KO mice had short, coiled, or absent flagella. $\mathbf{b}$ numerous defects in the sperm flagellar ultrastructures in the KO mice. The cross-section of flagella from KO mice presents disorganized arrangement of CP and ODF, and incomplete OD in the mid-piece. And the absence of OD and ODF were observed in the principal piece. (scale bars, $150 \mathrm{~nm}$ ), 
Table 2 Semen analysis in wild-type (WT) and knock-out (KO) male mice by CASA

\begin{tabular}{|c|c|c|}
\hline & \multicolumn{2}{|l|}{ Sample } \\
\hline & WT & ко \\
\hline \multicolumn{3}{|l|}{ Semen parameters } \\
\hline Sperm concentration $\left(10^{6} / \mathrm{ml}\right)^{\mathrm{a}, \mathrm{b}}$ & $26.59 \pm 3.56$ & $6.61 \pm 1.35$ \\
\hline Motility $(\%)^{b}$ & $73.36 \pm 6.22$ & $5.85 \pm 3.00$ \\
\hline Progressive motility $(\%)^{\mathrm{b}}$ & $72.07 \pm 6.44$ & $3.60 \pm 1.29$ \\
\hline \multicolumn{3}{|l|}{ Sperm locomotion parameters } \\
\hline Curvilinear velocity $(\mathrm{VCL})(\mu \mathrm{m} / \mathrm{s})^{\mathrm{b}}$ & $74.88 \pm 7.87$ & $11.60 \pm 1.71$ \\
\hline Straight-line velocity $(V S L)(\mu \mathrm{m} / \mathrm{s})^{b}$ & $15.80 \pm 3.60$ & $0.61 \pm 0.49$ \\
\hline Average path velocity $(\mathrm{VAP})(\mu \mathrm{m} / \mathrm{s})^{\mathrm{b}}$ & $31.27 \pm 4.55$ & $2.28 \pm 0.30$ \\
\hline $\begin{array}{l}\text { Amplitude of lateral head displacement } \\
(\mathrm{ALH})(\mu \mathrm{m})^{\mathrm{b}}\end{array}$ & $1.04 \pm 0.13$ & $0.22 \pm 0.01$ \\
\hline Linearity $(\text { LIN })^{b}$ & $0.21 \pm 0.04$ & $0.06 \pm 0.05$ \\
\hline Wobble $(\mathrm{WOB},=\mathrm{VAP} / \mathrm{VCL})^{\mathrm{b}}$ & $0.42 \pm 0.05$ & $0.20 \pm 0.05$ \\
\hline Straightness $(\mathrm{STR},=\mathrm{VSL} / \mathrm{VAP})^{\mathrm{b}}$ & $0.50 \pm 0.05$ & $0.25 \pm 0.17$ \\
\hline Beat-cross frequency $(B C F)(H z)^{b}$ & $7.57 \pm 1.14$ & $0.75 \pm 0.27$ \\
\hline
\end{tabular}

QRICH2 significantly inhibited the ubiquitination of exogenous AKAP3 and TSSK4 in the HEK 293 cells (Fig. 6f, g). These data suggest that QRICH2 is a key molecule that regulates sperm flagellar formation by stabilizing the related proteins through suppressing their ubiquitination degradation.

It has been suggested that ROPN1 and CABYR utilize docking interactions with AKAP3 to regulate cilia and flagellar formation through the PKA-related pathway ${ }^{27-29}$. In this study, we observed that the binding of Ropn1 and Cabyr to Akap3 was weakened in the KO mice compared with the WT mice by Co-IP assay (Fig. 6h). Furthermore, TSSK4 was reported to influence sperm flagellar formation by enhancing the phosphorylation state of ODF2, which is a prominent protein of the sperm tail outer dense fibers ${ }^{13,30-33}$. Because no commercial antibody was available, we did not confirm that TSSK4 can increase the phosphorylation of ODF2. However, we found that the overexpression of TSSK4 could increase ODF2 expression (Fig. 6i). In all, QRICH2 could suppress the ubiquitination degradation of AKAP3 and ROPN1 and facilitate CABYR expression as a trans-acting factor, correspondingly reinforcing the binding of AKAP3, ROPN1, and CABYR in sperm flagella. Additionally, QRICH2 regulates the formation of outer dense fiber in sperm flagella by directly enhancing the transcription of ODF2 and stabilizing TSSK4 through inhibition of its ubiquitination degradation, which is a crucial factor for ODF2 phosphorylation. Overall, these findings reveal that QRICH2 is required for maintaining the normal structure of sperm flagella by regulating key proteins in the related signaling pathways (Fig. 7a).

QRICH2 mutations detected in patients with asthenozoospermia. Two homozygous nonsense mutations of $\mathrm{QRICH} 2$ were observed in two MMAF patients from two consanguineous families. Additionally, Qrich2-deficient male mice showed MMAF phenotypes and infertility. These results suggested that QRICH2 plays a crucial role in male reproduction associated with MMAF. The results of proteomics analysis showed that several proteins which are not only related to the development of sperm tail structure, but also to energy metabolism were significantly reduced in Qrich2-deficient mice. The energy metabolism in sperm flagella provides the sliding force for flagella beating which directly affects the sperm motility. Reduced sperm motility is the basic character for asthenospermia which is defined as a semen sample containing less than $40 \%$ of motile sperm and less than $32 \%$ with progressive motility ${ }^{34}$. The expressions of ROPN1 and CABYR have been observed remarkably decreasing in asthenospermia subjects in comparison with the conrols ${ }^{35}$. Furthermore, the mutations of dynein genes were found in isolated nonsyndromic asthenozoospermia patients ${ }^{36}$. Consequently, we suppose that whether QRICH2 mutations are associated with asthenospermia. To investigate the variation of $Q R I C H 2$ in interfile males with asthenospermia, we sequenced all 18 exons and their flanking intronic regions of QRICH2 in 150 asthenospermia patients. Finally, six heterozygous mutations, including five missense mutations and one nonsense mutation, were observed in six subjects (Table 3). These mutations were predicted to be pathogenic by bioinformatic tools: SIFT ${ }^{37}$, PolyPhen$2^{38}$, and M-CAP 39 (Table 3). To further understand the effect of these mutations, we investigated the conformational changes of QRICH2 protein caused by these mutations with SWISSMODEL ${ }^{40}$ (https://swissmodel.expasy.org/) and PyMoL software $^{41}$ (1.3r1, DeLano Scientific LLC). The results of silico analysis showed that the mutations of c.3313C >A [p.Q1105K], c.3335G >A [p.G1112E], and c.4039A $>$ G [p.M1347V] could change the conformation of QRICH2 protein (Fig. 7b, d). Because no gap-free homology templates for the spacer fragments (aa 550-600 and aa 1300-1360) could be identified in the PDB database, the effects of c.1706T $>A$ [p.V569E] and c.4039A $>G$ [p.M1347V] on QRICH2 protein structure were not predicted.

Accordingly, no homozygous mutations or compound heterozygous mutations were found in the asthenospermia patients, suggesting that the recessive QRICH2 may just relate to MMAF. Several variants were indeed detected in the normal controls (200 normal fertility males) (Supplementary Table 1), whereas those rare and deleterious heterozygous mutations of QRICH2 were found only in asthenospermia (asthenozoospermia: 6/150; control: 0/200; $p<0.006$, the Fisher exact test, two-sided), indicating that deleterious heterozygous mutations of $\mathrm{QRICH} 2$ may increase the risk of asthenospermia. To further prove the vital role of heterozygous mutations of QRICH2 in sperm movement, we performed research on epididymal spermatozoa of $\mathrm{Ht}$ mice. We observed that the morphology of most sperm is normal in Ht mice (Supplementary Fig. 5b) and the number of abnormal sperm tail was not significantly different between $\mathrm{Ht}$ mice and WT mice (Fig. 7e). Notably, by CASA, the sperm motility parameters were decreased in Ht mice compared with WT mice (Supplementary Table 2). The diminished sperm movement in the Ht mice compared to WT mice is directly showed in Supplementary Movies 6 and 7. Given that the reduced sperm motility in Ht mice closely resembles the sperm phenotype of human asthenospermia, these data suggest that the deleterious heterozygous mutations of human QRICH2 may contribute to asthenozoospermia.

\section{Discussion}

Impaired sperm motility is a major contributor to male infertility, and the sperm flagellum is the key factor affecting sperm motility. Variations in the genes involved in the formation of sperm flagella can cause abnormalities in sperm flagellum morphology and further reduce sperm motility, thus impairing male fertility. Several genes have been reported to be associated with the development of the sperm flagellum in mouse models, whereas mutations in these genes in mice are rarely identified with those in humans with MMAF, except AKAP4, CCDC39, DNAH1, CFAP43, CFAP44, and CFAP69 (refs. ${ }^{4,5,8,16-19}$ ). Therefore, it is crucial to explore the pathogenic genes of MMAF in humans and further understand the mechanisms required for the structure of sperm tails and their effect on fertility. 
a

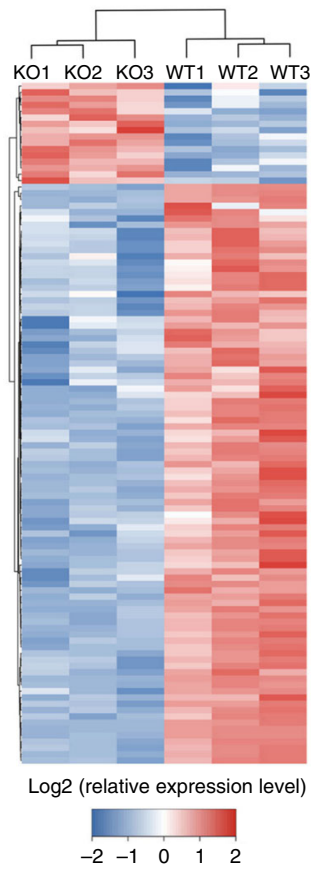

C
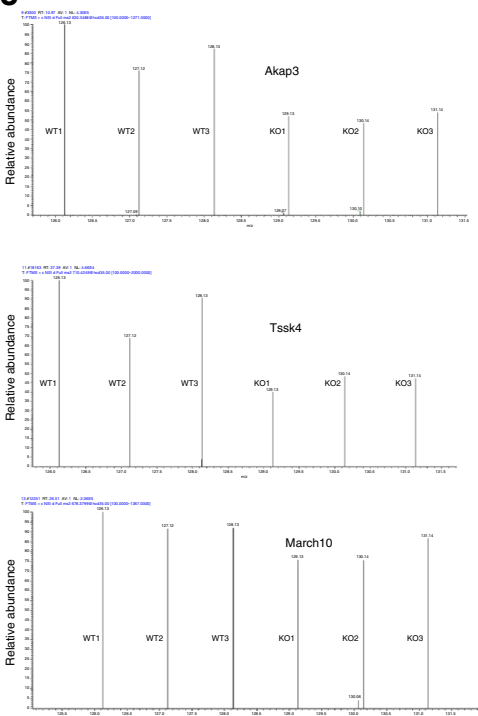

d

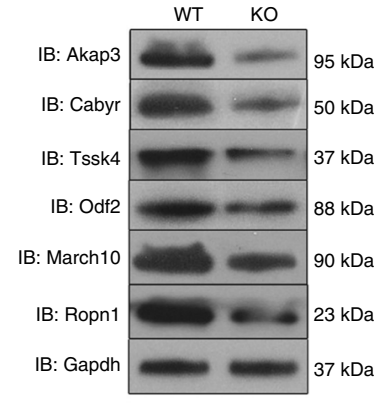

e b
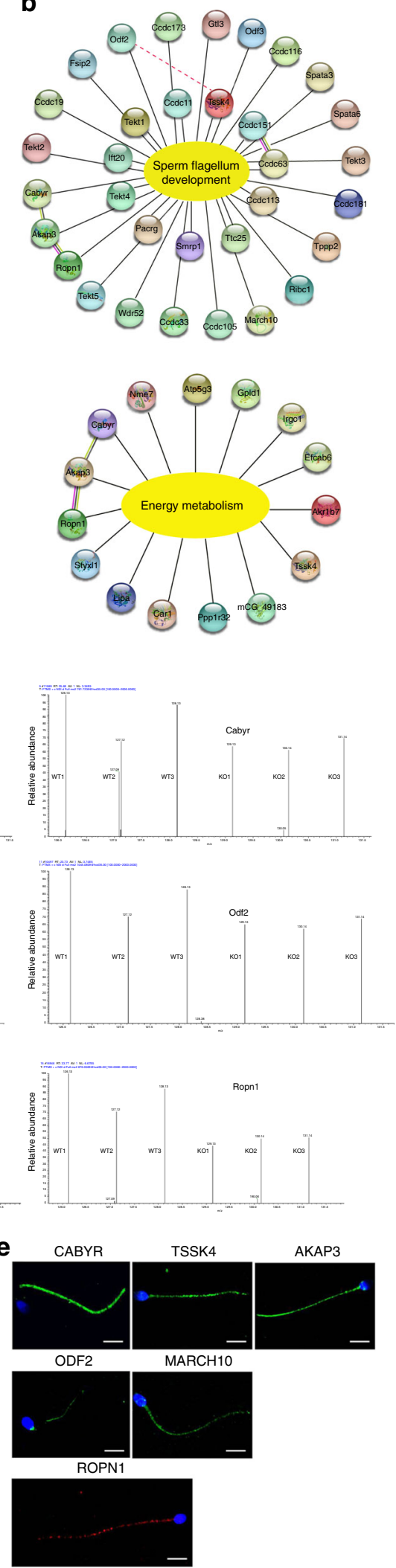

Fig. 5 Proteins for sperm flagellum development are down-regulated in $\mathrm{KO}$ mice. a The heat map from three independent proteomic analyses of testes from the WT and KO mice. $\mathbf{b}$ The proteins down-regulated in the KO mice are associated with sperm flagellum development and related energy metabolism. c The different MS/MS spectra of Akap3, Odf2, Tssk4, March10, Ropn1, and Cabyr in the KO mice and the WT mice. d The expression of the key proteins in the testes of the WT and KO mice. The western blotting results confirmed the decreased expression of Akap3, Odf2, Tssk4, March10, Ropn1 and Cabyr in the KO mice compared with the WT mice. ( $n=4$ biologically independent WT mice or KO mice). e The location of the vital proteins in human spermatozoa by immunofluorescence staining. AKAP3 (green), ODF2 (green), TSSK4 (green), MARCH10 (green), CABYR (green), and ROPN1 (red) are dominantly expressed in human spermatozoa flagella. (blue, DAPI; scale bars, $5 \mu \mathrm{m}$ ) 
a

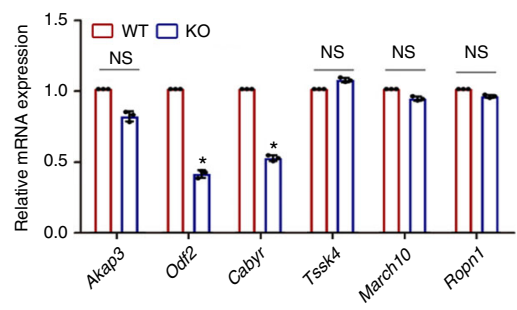

b

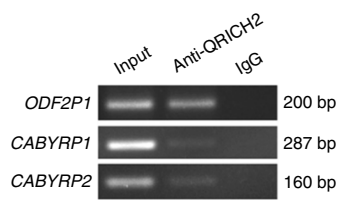

C

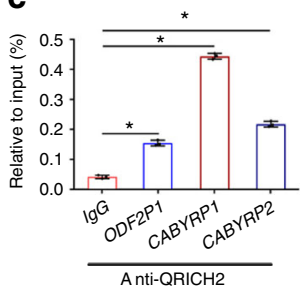

d

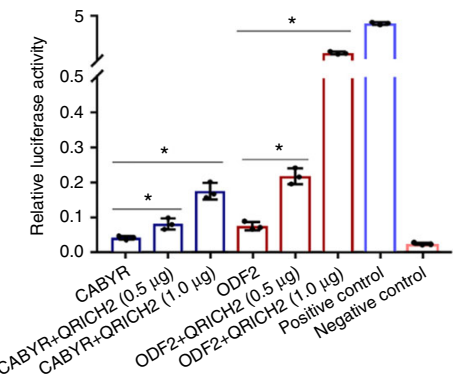

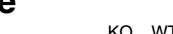
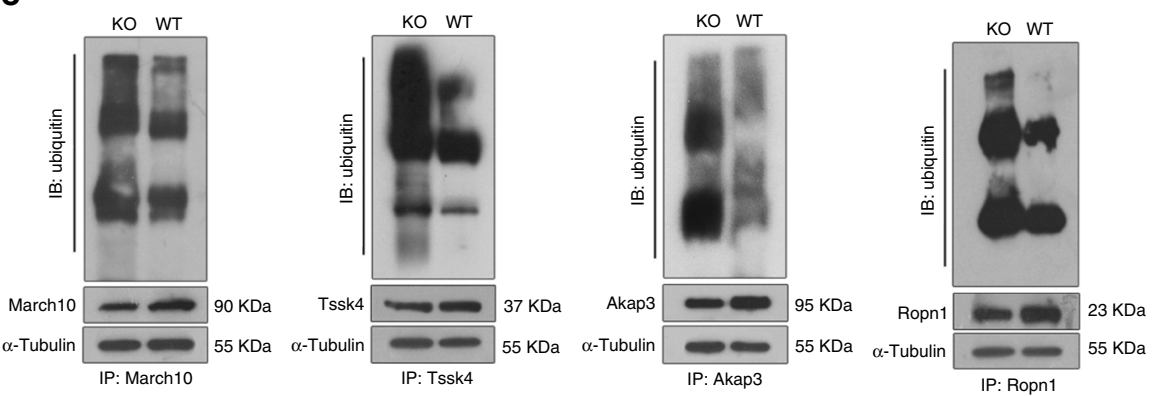

f

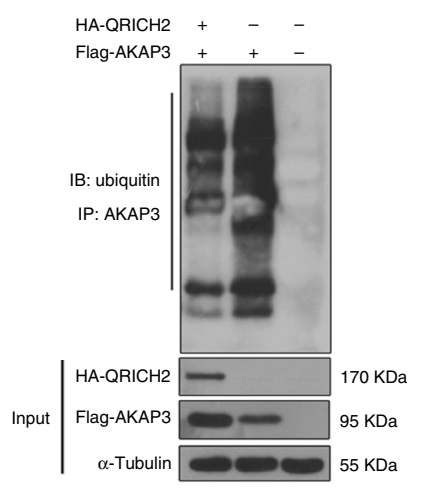

g

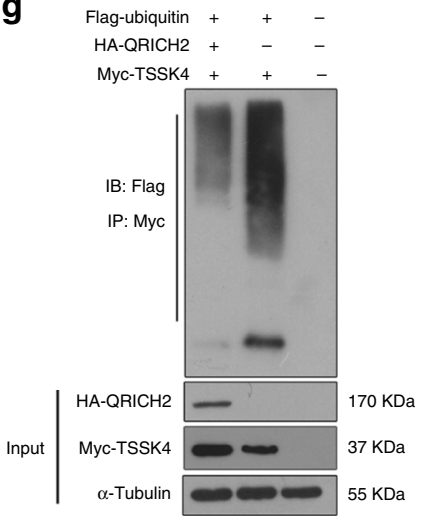

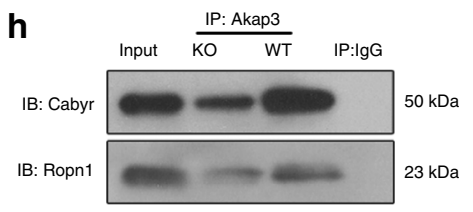

i

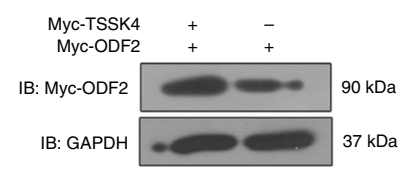

Fig. 6 The mechanisms of QRICH2 regulating sperm flagellar formation. a The mRNA levels of Akap3, Odf2, Tssk4, March10, Cabyr and Ropn1 in the testes of the KO and WT mice. The reduced mRNA levels of Odf2 and Cabyr in the KO mice were detected by real-time PCR (Student's $t$-test; $n=3$ independent experiments; ${ }^{*} p<0.05$; NS, not significant; error bars, s.e.m). b One QRICH2-occupied site in the ODF2 promoter and two QRICH2-occupied sites in the CABYR promoter were detected by ChIP-PCR assays of human testicular tissues. Input DNA and normal mouse IgG pulldowns were used as positive control and negative control, respectively. c ChIP-qPCR results showed the increased binding of QRICH2 to the ODF2 and CABYR promoter regions in the group with QRICH2 antibody compared to that with IgG (Student's $t$-test; $n=3$ independent experiments; " $p<0.05$; error bars, s.e.m). d QRICH2 could enhance the transcriptional activity of ODF2 and CABYR by luciferase reporter assays (Student's $t$-test; $n=3$ independent experiments; ${ }^{*} p<0.05$; error bars, s.e.m). e The increased ubiquitination levels of Akap3, Tssk4, Ropn1 and March10 in the testes of the KO mice compared with that in the WT mice. f, $\mathbf{g}$ QRICH2 decreased the ubiquitylation of exogenous AKAP3 (f) and TSSK4 ( $(\mathbf{g})$ in HEK 293 cells. The cells were harvested after a 6-h treatment with $10 \mu \mathrm{M}$ of MG132. $\mathbf{h}$ The binding of Ropn1 and Cabyr to Akap3 was weakened in the testes of the KO mice compared with that in the WT mice. i Overexpression of TSSK4 could increase the protein levels of ODF2

In this study, we detected two homozygous nonsense mutations of the QRICH2 in two MMAF patients from two consanguineous families. It is the first time that QRICH2 has been identified to cause MMAF in humans, providing a new genetic diagnostic cue for male infertility. Our further investigations suggest that the two variants result in dysplasia of sperm flagellum: almost all observed sperm from affected patients exhibited multiple morphological abnormalities of the sperm flagella. Additionally, irregular sperm ultrastructures were observed by TEM: peripheral fibers or microtubules were absent, such as in $9+0$ and $6+0$ axonemal arrangement. Furthermore, the Qrich2-deficient mouse models showed MMAF phenotypes and abnormal flagellum ultrastructure, supporting the vital role of QRICH2 in the development of the sperm flagellum and that homozygous loss-of-function mutations in QRICH2 cause the abnormal development of the sperm flagellum, which leads to male infertility.
QRICH2 is evolutionarily conserved in several species, indicating its fundamental role in organisms. However, the functions of QRICH2 have not been characterized so far. QRICH2 contains three principal domains: Glutenin hmw super family, SMC_N super family and DUF4795 (https://www.ncbi.nlm.nih.gov). The SMC_N super family is the only known functional domain in QIRCH2. The structural maintenance of chromosomes (SMC) super family proteins have ATP-binding domains at the $\mathrm{N}$-termini and C-termini, which are involved in chromatin and DNA dynamics ${ }^{42-44}$. SMC-like domains are highly conserved in some ciliary and centrosomal proteins, which are related to intraflagellar transport ${ }^{45-48}$, suggesting that SMC-like domains might represent signature sequences that are recognized during these transport processes. Importantly, the mutations in the SMC_N domain of CFAP43 and CCDC39 have been reported to be associated with $\mathrm{MMAF}^{8,18}$. Hence, QRICH2 containing the SMC_N 
a

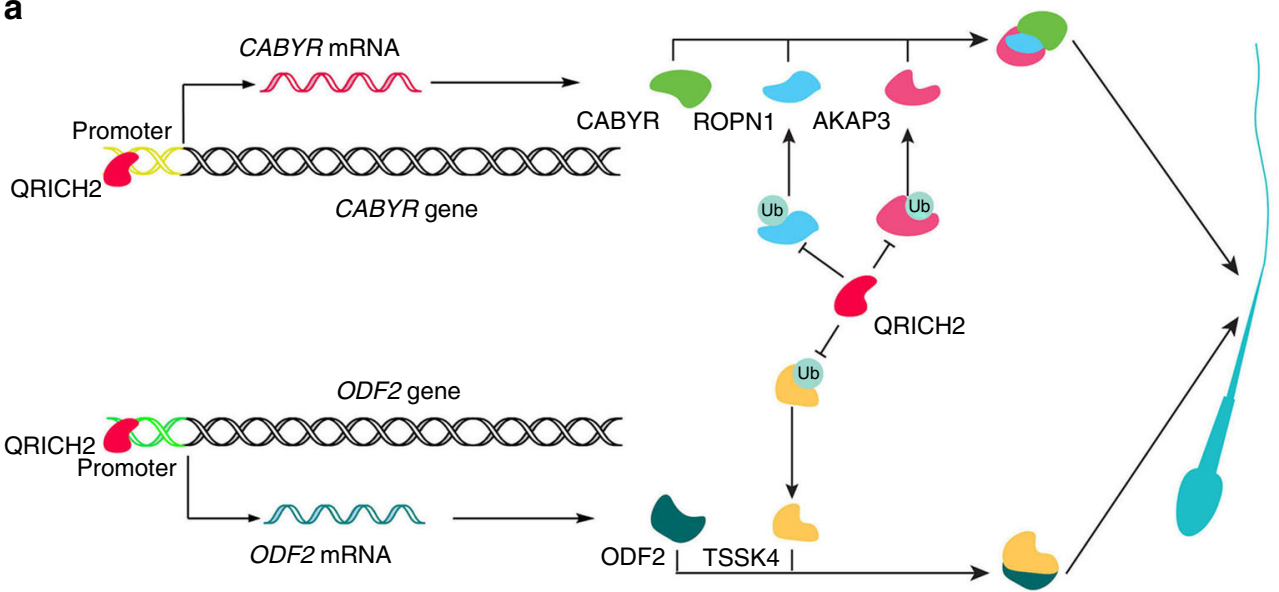

b

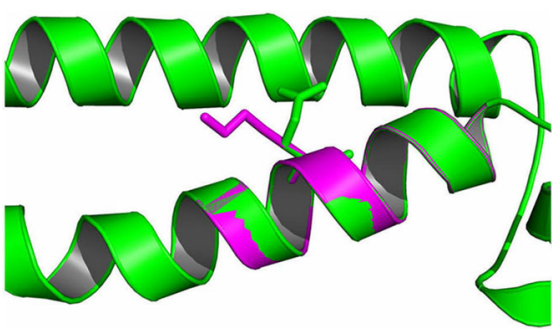

C

d
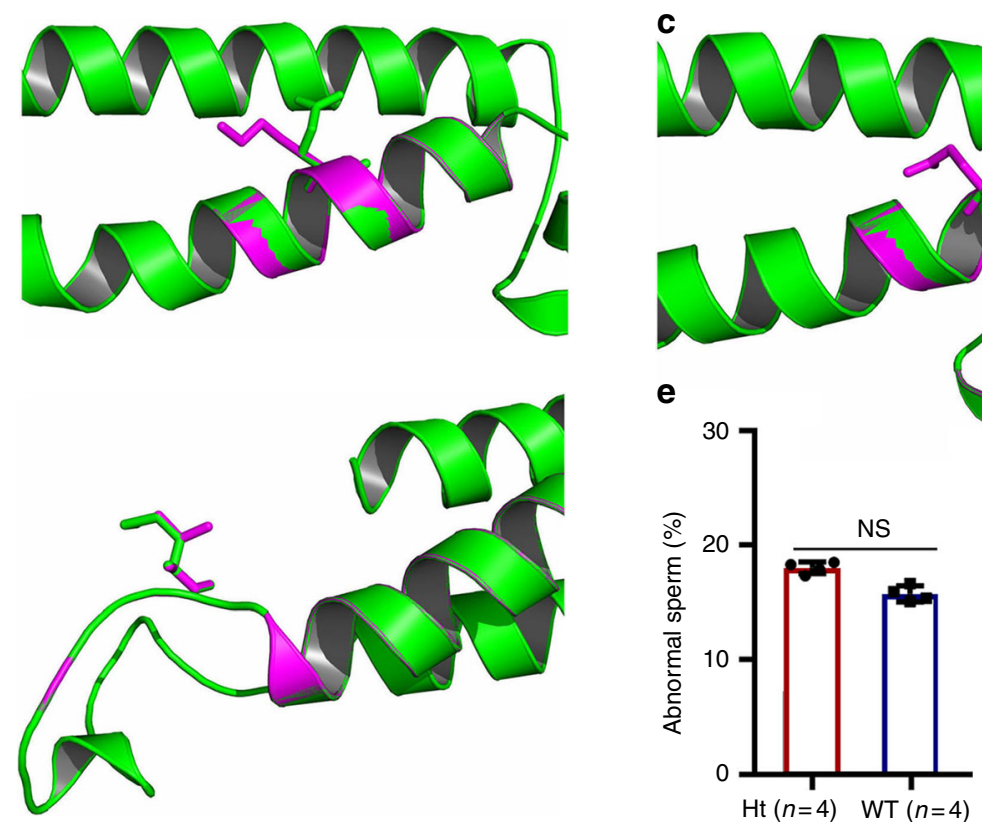

Fig. 7 The mechanisms were elucidated by proposed models. a The mechanisms of QRICH2 modulating the development of sperm flagella. QRICH2 promoted sperm flagellar formation through upregulating ODF2 and CABYR expression as a transcription factor, inhibiting ubiquitin-mediated degradation of AKAP3, TSSK4, and ROPN1 and finally reinforcing the interaction of AKAP3/CABYR/ROPN1 and TSSK4/ODF2 during the biogenesis of sperm flagella. b-d Effects of QRICH2 mutations on the structural conformation of QRICH2 protein. Superimposed structures of wild type (green) and mutant (purple) of QRICH2. The structures of QRICH2 protein were changed by the mutations of c.3313C>A [p.Q1105K] (b), c.3335G>A [p.G1112E] (c) and c.4039A>G [p.M1347V] (d). e The difference of the abnormal sperm tail number between WT mice and Ht mice was not significant. (Student's $t$-test; $n=3$ biologically independent WT mice or Ht mice; NS not significant; error bars, s.e.m)

Table 3 Analysis of QRICH2 mutations in asthenozoospermia patients

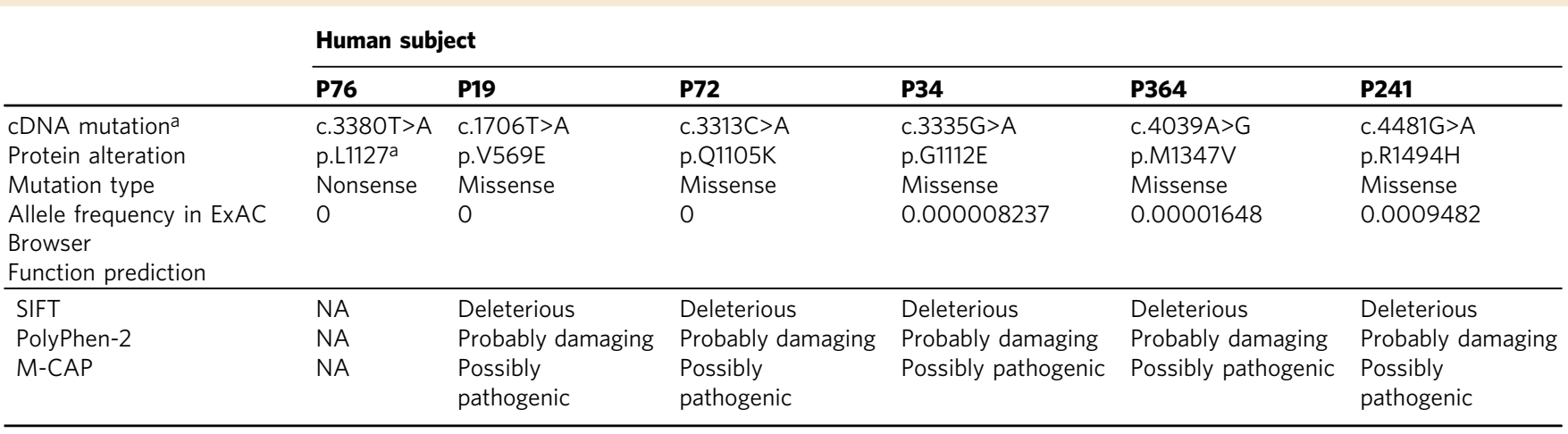

NA not available

aThe accession number for QRICH2 is GenBank: NM_032134.2 
domain supports that QRICH2 is required for sperm flagellar development.

QRICH1 is the homologous gene of QRICH2, whose mutations are associated with developmental delay ${ }^{49,50}$. However, to date, there are no studies reporting the functions of $\mathrm{QRICH} 2$. To understand the molecular mechanism of QRICH2 in the development of the sperm flagella, we used a proteomics approach to compare the different expression of proteins in the testes of the $\mathrm{WT}$ and $\mathrm{KO}$ males. Intriguingly, 33 proteins suggested to be associated with the formation of sperm tail were significantly decreased in the KO mice compared with the WT mice ${ }^{9,51-54}$. Among them, CFAP44 variants have been reported to cause MMAF in humans ${ }^{8,16}$. Mice missing some of these genes have demonstrated abnormal sperm tail structures (Spata6, Tekt4, Odf2, Ropn1, and Cabyr) ${ }^{10-15}$. A potential mechanism is that ROPN1 and CABYR are involved in the development of the sperm flagella though binding with AKAP3 and further regulating PKA signaling 28,29 . In this study, we observed that QRICH2 inhibited the ubiquitination degradation of AKAP3 and ROPN1, increased CABYR mRNA levels, and further maintained the complex of AKAP3, to which ROPN1 and CABYR stably bind in the sperm flagella. Meanwhile, QRICH2 stabilized TSSK4/ODF2mediated pathways, which are crucial for sperm tail structure growth $^{12,13,30-33}$, by promoting their protein and mRNA expression. In addition, QRICH2 restrained the ubiquitination degradation of MARCH10, a microtubule-associated E3 ubiquitin ligase regulating the formation and maintenance of the flagella in developing spermatids. Our findings elucidate the potential mechanisms of QRICH2 in regulating the development of the sperm flagellum and provide additional cues for future research on the functions of QRICH2.

In this study, no homozygous mutations but six deleterious heterozygous variants were detected in patients with asthenozoospermia. Remarkably, in addition to the deleterious heterozygous variant (c.3313C>A), P72 carried a rare SNP (rs780426606). For P241, two other SNPs (rs67948583 and rs73999020) with extremely low frequency were determined. We therefore speculate that the compound deleterious heterozygous variants and rare SNPs of QRICH2 may be the risk for P72 and P241. Besides that, the Ht mice represented the decreased sperm motility. Thus so, the pathologic heterozygous variants of $\mathrm{QRICH} 2$ could increase the risk for asthenozoospermia. Certainly, this theory needs to be further investigated.

In summary, our genetic and functional data based on human subjects and mouse models strongly suggest that homozygous loss-of-function mutations of $\mathrm{QRICH} 2$ are a novel genetic cause of MMAF. Additionally, the underlying mechanism that QRICH2 regulates sperm flagellar development was elucidated in this study. The screening of the deleterious mutations of QRICH2 could be important for clinical molecular diagnosis of male infertility. As the findings about QRICH2 in this study are novel, further research is required on this unfamiliar gene to explore the importance of QRICH2 in male fertility.

\section{Methods}

Study participants. Two interfile males with MMAF from two consanguineous families were enrolled at the Human Sperm Bank of West China Second University Hospital of Sichuan University and the Center of Reproductive Medicine at the First Affiliated Hospital of Nanjing Medical University. Family members of these patients were additionally recruited. Furthermore, 150 patients of Han ethnicity with primary infertility and diagnosed with asthenozoospermia (the percentage of progressively motile sperm $<32 \%$ ) from the Department of Medical Genetics, the Department of Urology in West China Hospital, Sichuan University were enrolled for the population study. Patients were excluded any significant androgenic or endocrine abnormalities in a physical examination, hormone analysis and scrotal ultrasound, including hypogonadotropic hypogonadism, cryptorchidism, varicocele, seminal ductal obstruction, testicular trauma and tumor. The patients have chromosomal aberration were also excluded. The detailed sperm parameters of the patients were listed in Supplementary Data 2. A total of 200 unrelated Han Chinese males with normal fertility (fathering at least one offspring by natural fertilization) and sperm quality (sperm concentration $\geq 15 \mathrm{million} / \mathrm{ml}$; the percentage of progressively motile sperm $\geq 32 \%$ ) were recruited from volunteers served as the control group for the population study. The inclusion and exclusion criteria used in this study were selected according to previously published reports and the guidelines of the World Health Organization ${ }^{4-6,34,55}$. The study was approved by the Ethical Review Board of West China Second University Hospital, Sichuan University. Informed consent was obtained from each subject in our study.

Whole-exome sequencing (WES) and Sanger sequencing. Genomic DNA was isolated from peripheral-blood samples of the subjects using a whole blood DNA purification kit (QIAGEN). Whole-exome sequencing (WES) was performed on the two MMAF patients from two consanguineous families. $1 \mu \mathrm{g}$ of genomic DNA was utilized for exon capture using the Agilent SureSelect Human All Exon V6 Kit and sequenced on the Illumina HiSeq X system according to the manufacturer's instructions. The average sequencing depth on target was 108.11, and the ratio of target fraction covered at minimum $10 \times$ was $99.4 \%$.

Reads were mapped to the reference genome (UCSC hg19) by the BurrowsWheeler Aligner (BWA) software to get the original mapping result in BAM format, and duplicates were marked and removed using Picard (http:// broadinstitute.github.io/picard/index.html). ANNOVAR was performed for functional annotation through a variety of databases, such as dbSNP, 1000 Genomes Project, ExAC and HGMD. After filtering, the retained nonsynonymous SNVs were submitted to PolyPhen-2, SIFT, MutationTaster and CADD for functional prediction.

Candidate pathogenic variants in the family members and 18 coding exons and their flanking intronic regions of QRICH2 in the unrelated population were validated by Sanger sequencing. PCR amplification was performed with Dyad Polymerase (Bio-Rad Laboratories). DNA sequencing of PCR products was conducted on an ABI377A DNA sequencer (Applied Biosystems). The primers for PCR are listed in Supplementary Table 3.

Tandem Mass Tagging (TMT) proteomics analysis. The primary experimental procedures for TMT proteomics analysis include protein preparation, trypsin digestion, TMT labeling, HPLC fractionation, LC-MS/MS analysis and data analysis. The detailed procedure is presented in the Supplementary methods. The TMT proteomics analysis in our research is supported by Jingjie PTM BioLabs.

Generation of Qrich2-deficient mouse models. All animal experiments were performed in accordance with the recommendation of the Guide for the Care and Use of Laboratory Animals of the National Institutes of Health. The animal experiments were approved by the Experimental Animal Management and Ethics Committee of West China Second University Hospital, and all animal works were performed in accordance with the guidelines and regulations the Committee. The frameshift mutation was generated using the CRISPR-Cas9 technology by the nonhomolog recombination method. The guide RNAs were designed against the 5'UTR and intron 2 of Qrich2. The Cas 9 mRNA and gRNA were obtained by in vitro transcription assay and then microinjected into the cytoplasm of single-cell C57BL/6J embryos. The injected embryos were transferred into the oviducts of pseudopregnant mice. The frameshift mutation in Qrich2 was identified in the founder mice and their offspring by PCR, real-time PCR and western blotting. The primers for PCR and real-time PCR are listed in Supplementary Table 3 and Table 4.

Plasmid construction. The full-length cDNA of AKAP3, TSSK4, ODF2, and QRICH2 was synthesized and separately cloned into pENTER (Vigene), pCMV6 Entry (Origene), pCMV6-Entry, and $\mathrm{pcDNA}^{\mathrm{TM}_{3.1}{ }^{(+)}}$(Invitrogen) vectors containing a FLAG, Myc, and HA tag sequence. CABYR and ODF2 promoter fragments were amplified and cloned into the pGL3-Basic luciferase reporter vector (Promega). The shRNAs designed to interfere with QRICH2 expression and the negative control SuperSilencing shRNA (shNC) were synthesized and cloned into the psi-LVRU6GP vector by GeneCopoeia. The target sequences of QRICH2 shRNAs were 5'-GGGTTCACTTCCTTAACATCA-3'. The primers for promoter region amplification are listed in Supplementary Table 5.

Real-time PCR. Total RNA of mice tissues was extracted using TRIzol reagent (Invitrogen) and was converted to cDNA using a RevertAid First-Strand cDNA Synthesis Kit (ThermoFisher). Real-time PCR was performed using SYBR Premix Ex Taq II (TaKaRa) on an iCycler RT-PCR Detection System (Bio-Rad Laboratories). The $\Delta \Delta$ CT method was used for data analysis ${ }^{56}$. Each assay was performed in triplicate for each sample. The Gapdh gene was used as an internal control. The primers for real-time PCR are listed in Supplementary Table 4.

Dual-luciferase reporter assays. Two plasmids, including the pGL3-ODF2 promoter region and pGL3-CABYR promoter region, were separately co-transfected with the HA-QRICH2 plasmid and individually transfected into HEK 293 cells, with the pGL3-control plasmid as the positive control and the pGL3-basic plasmid 
as the negative control. After $48 \mathrm{~h}$, the luciferase activity of the cell lysates was analyzed using a dual-luciferase reporter assay system (Promega).

Antibodies. The antibodies used in western blotting, Co-IP and immunofluorescence staining are as follows: anti-QRICH2 (1:200, sc-514279, Santa Cruz Biotechnology), anti-QRICH2 (1:50, HPA052219, Sigma-Aldrich) for immunofluorescence staining of mice sample, anti-QRICH2 (1:50, HPA021935, SigmaAldrich) for immunofluorescence staining of human sample, anti-AKAP3 (1:100, provided by Prof. Huayu Qi) for immunofluorescence staining, anti-AKAP3 (1:100, 13907-1-AP, Proteintech) for Co-IP, anti-ODF2 (1:500, 12058-1-AP, Proteintech), anti-CABYR (1:500, 12351-1-AP, Proteintech), anti-ROPN1 (1:200, sc-130455, Santa Cruz Biotechnology), MARCH10 (1:500, bs-10732R, Bioss), antiTSSK4 (1:400, A7861, ABclonal), anti-ubiquitin (1:1000, ab7780, Abcam), anti-Flag Tag (1:500, 66008-2-Ig, Proteintech), anti-Myc Tag (1:1000, sc-764, Santa Cruz Biotechnology), anti- $\alpha$-Tubulin (1:2000, T7451, Sigma-Aldrich) and anti-GAPDH (1:5000, ab8245, Abcam). Anti-AKAP3 mouse monoclonal antibody for western blotting was kindly provided by Prof. Huayu Qi. Anti-TSSK4 rabbit polyclonal antibody for Co-IP was kindly provided by Prof. Long Yu.

Western blotting. The proteins of the cultured cells and mice testes tissues were extracted using a universal protein extraction lysis buffer (Bioteke) containing a protease inhibitor cocktail (Roche). The denatured proteins were separated on $10 \%$ SDS-polyacrylamide gels and transferred to a polyvinylidene difluoride (PVDF) membrane (Millipore) for the immunoblot analysis.

Co-immunoprecipitation (Co-IP). The extracted proteins were incubated with $3 \mu \mathrm{g}$ of target antibodies overnight at $4{ }^{\circ} \mathrm{C}$. Next, $50 \mu \mathrm{l}$ of Protein A/G magnetic beads (Invitrogen) was added to each incubation sample for $1 \mathrm{~h}$ at room temperature. The beads were washed three times with $1 \times$ PBS. Finally, the coimmunoprecipitated proteins were eluted by standard $1 \times$ SDS sample buffer and heated for $10 \mathrm{~min}$ at $70^{\circ} \mathrm{C}$ and then separated on $10 \%$ SDS-polyacrylamide gels and PVDF membranes for the immunoblot analysis.

Chromatin immunoprecipitation (ChIP)-PCR and ChIP-qPCR. ChIP assays were performed using a ChIP-IT Express Enzymatic Kit (Active Motif). In brief, the testes tissues were fixed with $1 \%$ formaldehyde, and chromatin was extracted in cell lysis buffer and then in nuclear lysis buffer with protease inhibitor cocktail and then sheared by sonication (Bioruptor, Scientz) on ice to a mean length of $500 \mathrm{bp}$. $1 \%$ of the sheared chromatin was retained as a positive control input DNA and another portion was precipitated by incubation with IgG as a negative control in the subsequent PCRs. The remaining chromatin was precipitated by incubation with antibodies against QRICH2. PCR and qPCR were performed to amplify the target regions of $C A B Y R$ and ODF2 which QRICH2 binding to. The qPCR data were analyzed by Fold Enrichment Method ${ }^{57}$ and Percent Input Method ${ }^{58}$ simultaneously, and in Percent Input Method, the value of target DNA fragments that were enriched in testes tissue was normalized to the value of the $1 \%$ input DNA. Each assay was performed in triplicate to confirm the reproducibility of the results. The primers are listed in Supplementary Table 3.

Flow cytometric analysis and cell sorting. The testes from 8-week-old male mice were removed and decapsulated. Firstly, the seminiferous tubules were incubated in $10 \mathrm{ml} 1 \times$ PBS containing $90 \mathrm{mg} / \mathrm{ml}$ of collagenase (Invitrogen) with continuous agitation for $15 \mathrm{~min}$ at $32^{\circ} \mathrm{C}$ and then allowed to sediment for $5 \mathrm{~min}$ and the supernatant was removed. Next, the pellet was resuspended in $10 \mathrm{ml}$ of PBS with $60 \mathrm{mg} / \mathrm{ml}$ of trypsin (Sigma-Aldrich) and $1 \mu \mathrm{g} / \mathrm{ml}$ of DNase (Promega), and incubated under the same conditions for $15 \mathrm{~min}$. After gently being pipetted with a Pasteur pipette, the cell suspension was centrifuged at $400 \times g$ for $10 \mathrm{~min}$ and then washed three times with $1 \times$ PBS, filtered using $40 \mu \mathrm{m}$ nylon mesh to remove cell clumps and last resuspended in HEPES-buffered RPMI containing $0.5 \%$ BSA. After the testicular single cell suspensions were obtained, two million cells were diluted in $2 \mathrm{ml}$ of $1 \times$ PBS buffer and stained with Hoechst $33342(5 \mu \mathrm{g} / \mathrm{ml}$; Sigma) for $1 \mathrm{~h}$ at $32^{\circ} \mathrm{C}$. Before analysis, PI $(2 \mu \mathrm{g} / \mathrm{ml}$; Sigma $)$ was added to exclude dead cells. Finally, cell analysis and sorting were performed on a FACScalibur flow cytometer (Beckman Coulter, CA, USA) equipped with a cell sorting system.

Immunofluorescence staining. After being washed with Sperm Washing Medium (CooperSurgical Inc.), the spermatozoa samples or the selected germ cells were fixed onto slides with $4 \%$ paraformaldehyde, permeabilized with $0.5 \%$ Triton $\mathrm{X}-100$, and blocked with $1 \%$ BSA. Next, the slides were sequentially incubated with primary antibodies overnight at $4{ }^{\circ} \mathrm{C}$. The slides were washed in $1 \times \mathrm{PBS}$, incubated with Alexa Fluor 488 (1:1000, A21206, Thermo Fisher) or Alexa Fluor 594 (1:1000, A11005, Thermo Fisher) labeled secondary antibodies for $1 \mathrm{~h}$ at room temperature, and then counterstained with 4,6-diamidino-2-phenylindole (DAPI, SigmaAldrich) to label the nuclei. Images were acquired using a laser scanning confocal microscope (Olympus).
Histology hematoxylin-eosin (H\&E) staining. Testicular and epididymal tissue samples from male mice were fixed with $4 \%$ paraformaldehyde overnight, dehydrated in ethanol, embedded in paraffin, and sectioned at $5 \mu \mathrm{m}$. The sections were stained routinely with hematoxylin and eosin for histological examination.

Electron microscopy. For scanning electron microscopy (SEM) assay, the spermatozoa samples were fixed onto slides of $1 \mathrm{~cm}$ diameter using $2.5 \%$ glutaraldehyde for $4 \mathrm{~h}$ at $4^{\circ} \mathrm{C}$. After washing the slides with $1 \times$ PBS three times and post-fixing in $1 \%$ osmic acid for $1 \mathrm{~h}$ at $4{ }^{\circ} \mathrm{C}$, dehydration was performed using $30,50,75,95$, and $100 \%$ ethanol sequentially, and the slides were dried using a $\mathrm{CO}_{2}$ critical-point dryer (Eiko HCP-2, Hitachi). Finally, the slides were mounted on aluminum stubs, sputter-coated by an ionic sprayer meter (Eiko E-1020, Hitachi), and analyzed by SEM (Hitachi S3400) under an accelerating voltage of $10 \mathrm{kV}$.

For the transmission electron microscopy (TEM) assay, each semen sample was rinsed in Sperm Washing Medium. The semen samples were pre-fixed in 3\% glutaraldehyde, post-fixed in $1 \%$ buffered OsO4, dehydrated through gradient acetone solutions, and embedded in Epon 812. Before ultrathin-sectioning, a halfthin section was made to enable sperm location under a light microscope. The ultrathin sections were double-stained with both lead citrate and uranyl acetate and then analyzed under a TEM (TECNAI G2 F20, Philips) with an accelerating voltage of $80 \mathrm{kV}$.

STORM imaging. Sperm cells were fixed and immunostained with rabbit antiQRICH2 (1:50, HPA021935, Sigma-Aldrich) and mouse anti- $\alpha$-Tubulin (1:100, T7451, Sigma-Aldrich) overnight at $4{ }^{\circ} \mathrm{C}$. After washed in $1 \times \mathrm{PBS}$, sperm cells were incubated with Atto 488 (1:500, 41051, Sigma-Aldrich) or Alexa fluor 568 (1:500 A10037, Thermo Fisher) secondary antibodies for $1 \mathrm{~h}$ at room temperature. Finally, the sperm samples were stained with imaging buffer (PBS with the addition of $100 \mathrm{mM}$ mercaptoethylamine at $\mathrm{pH} 8.5,5 \%$ glucose $(\mathrm{w} / \mathrm{v}), 0.5 \mathrm{mg} / \mathrm{ml}$ glucose oxidase (Sigma-Aldrich), and $40 \mu \mathrm{g} / \mathrm{ml}$ catalase (Roche Applied Science)) and illuminated at $657 \mathrm{~nm}$ for imaging. The $501 \mathrm{~nm}$ and $568 \mathrm{~nm}$ lasers were used to activated Atto 488, and Alexa fluor 568, respectively. A cylindrical lens (focal length $=1 \mathrm{~m}$ ) used for $3 \mathrm{D}$ localization was inserted into the detection path to enable determination of $\mathrm{z}$ positions from the ellipticities of the molecular images and the $x$ and $y$ positions from the centroid positions.

Statistical analysis. The statistical analyses were performed using the SPSS 17.0 software (IBM Company). Student's $t$-test and Fisher exact test were used to compare the observed indexes between the experimental groups. The $P$ values less than 0.05 were considered significant.

Reporting summary. Further information on experimental design is available in the Nature Research Reporting Summary linked to this article.

\section{Data availability}

Data on proteomic analysis described here are available on PRIDE 'PXD011730'. All relevant data that support the findings of this study are available from the corresponding author upon reasonable request.

Received: 9 April 2018 Accepted: 14 December 2018

Published online: 25 January 2019

\section{References}

1. Haxton, M., Fleming, R. \& Coutts, J. Population study of causes, treatment, and outcome of infertility. Br. Med. J. 292, 272 (1986).

2. Hull, M. et al. Population study of causes, treatment, and outcome of infertility. Br. Med. J. 291, 1693-1697 (1985).

3. Curi, S. et al. Asthenozoospermia: analysis of a large population. Arch. Androl. 49, 343-349 (2003)

4. Coutton, C., Escoffier, J., Martinez, G., Arnoult, C. \& Ray, P. F. Teratozoospermia: spotlight on the main genetic actors in the human. Hum. Reprod. Update 21, 455-485 (2015).

5. Khelifa, M. B. et al. Mutations in DNAH1, which encodes an inner arm heavy chain dynein, lead to male infertility from multiple morphological abnormalities of the sperm flagella. Am. J. Hum. Genet. 94, 95-104 (2014)

6. Yang, S. M. et al. Morphological characteristics and initial genetic study of multiple morphological anomalies of the flagella in China. Asian J. Androl. 17, 513 (2015).

7. Sil flow, C. D. \& Lefebvre, P. A. Assembly and motility of eukaryotic cilia and flagella. Lessons from Chlamydomonas reinhardtii. Plant Physiol. 127, 1500-1507 (2001) 
8. Tang, S. et al. Biallelic mutations in CFAP43 and CFAP44 cause male infertility with multiple morphological abnormalities of the sperm flagella. Am. J. Hum. Genet. 100, 854-864 (2017).

9. Lehti, M. S. \& Sironen, A. Formation and function of sperm tail structures in association with sperm motility defects. Biol. Reprod. 97, 522-536 (2017).

10. Yuan, S. et al. Spata6 is required for normal assembly of the sperm connecting piece and tight head-tail conjunction. Proc. Natl Acad. Sci. 112, E430-E439 (2015).

11. Roy, A., Lin, Y. N., Agno, J. E., DeMayo, F. J. \& Matzuk, M. M. Absence of tektin 4 causes asthenozoospermia and subfertility in male mice. FASEB J. 21, 1013-1025 (2007).

12. Wang, X. et al. Tssk4 is essential for maintaining the structural integrity of sperm flagellum. MHR: Mol. Hum. Reprod. 21, 136-145 (2014).

13. Donkor, F. F., Mönnich, M., Czirr, E., Hollemann, T. \& Hoyer-Fender, S Outer dense fibre protein 2 (ODF2) is a self-interacting centrosomal protein with affinity for microtubules. J. Cell Sci. 117, 4643-4651 (2004).

14. Fiedler, S. E., Dudiki, T., Vijayaraghavan, S. \& Carr, D. W. Loss of R2D2 proteins ROPN1 and ROPN1L causes defects in murine sperm motility, phosphorylation, and fibrous sheath integrity. Biol. Reprod. 88, 41-41 (2013).

15. Young, S. A. et al. CABYR is essential for fibrous sheath integrity and progressive motility in mouse spermatozoa. J. Cell. Sci. 129, 4379-4387 (2016).

16. Coutton, C. et al. Mutations in CFAP43 and CFAP44 cause male infertility and flagellum defects in Trypanosoma and human. Nat. Commun. 9, 686 (2018).

17. Baccetti, B. et al. Gene deletions in an infertile man with sperm fibrous sheath dysplasia. Hum. Reprod. 20, 2790-2794 (2005).

18. Merveille, A. C. et al. CCDC39 is required for assembly of inner dynein arms and the dynein regulatory complex and for normal ciliary motility in humans and dogs. Nat. Genet. 43, 72 (2011).

19. Dong, F. N. et al. Absence of CFAP69 Causes Male Infertility due to Multiple Morphological Abnormalities of the Flagella in Human and Mouse. Am. J. Hum. Genet. 18, 30096-X (2018).

20. Huang, B., Wang, W., Bates, M. \& Zhuang, X. Three-dimensional superresolution imaging by stochastic optical reconstruction microscopy. Science 319, 810-813 (2008).

21. Rust, M. J., Bates, M. \& Zhuang, X. Sub-diffraction-limit imaging by stochastic optical reconstruction microscopy (STORM). Nat. Methods 3, 793-795 (2006).

22. Xu, K., Babcock, H. P. \& Zhuang, X. Dual-objective STORM reveals threedimensional filament organization in the actin cytoskeleton. Nat. Methods $\mathbf{9}$, 185-188 (2012).

23. Vaughan, J. C., Jia, S. \& Zhuang, X. Ultrabright photoactivatable fluorophores created by reductive caging. Nat. Methods 9, 1181-1184 (2012).

24. Satir, P. \& Christensen, S. T. Overview of structure and function of mammalian cilia. Annu. Rev. Physiol. 69, 377-400 (2007).

25. Eddy, E. M., Toshimori, K. \& O'Brien, D. A. Fibrous sheath of mammalian spermatozoa. Microsc. Res. Tech. 61, 103-115 (2003).

26. Amaral, A. et al. Human sperm tail proteome suggests new endogenous metabolic path-ways. Mol. Cell Proteom. 12, 330-342 (2013).

27. Newell, A. E. H. et al. Protein kinase A RII-like (R2D2) proteins exhibit differential localization and AKAP interaction. Cytoskeleton 65, 539-552 (2008).

28. Li, Y. F. et al. CABYR isoforms expressed in late steps of spermiogenesis bind with AKAPs and ropporin in mouse sperm fibrous sheath. Reprod. Biol. Endocrinol. 8, 101 (2010).

29. Li, Y. F. et al. CABYR binds to AKAP3 and Ropporin in the human sperm fibrous sheath. Asian J. Androl. 13, 266 (2011).

30. Wang, X. et al. Testis-specific serine/threonine protein kinase 4 (Tssk4) phosphorylates Odf2 at Ser-76. Sci. Rep. 6, 22861 (2016).

31. Brohmann, H., Pinnecke, S. \& Hoyer-Fender, S. Identification and characterization of new cDNAs encoding outer dense fiber proteins of rat sperm. J. Biol. Chem. 272, 10327-10332 (1997).

32. Shao, X., Tarnasky, H. A., Schalles, U., Oko, R. \& van der Hoorn, F. A. Interactional Cloning of the 84-kDa Major Outer Dense Fiber Protein Odf84 LEUCINE ZIPPERS MEDIATE ASSOCIATIONS OF Odf84 AND Odf27. J. Biol. Chem. 272, 6105-6113 (1997).

33. Schalles, U., Shao, X., van der Hoorn, F. A. \& Oko, R. Developmental expression of the $84-\mathrm{kDa}$ ODF sperm protein: localization to both the cortex and medulla of outer dense fibers and to the connecting piece. Dev. Biol. 199, 250-260 (1998).

34. World Health Organization. WHO Laboratory Manual for the Examination and Processing of Human Sperm. (World Health Organization, Geneva, Switzerland, 2010).

35. Pelloni, M. et al. Molecular study of human sperm RNA: Ropporin and CABYR in asthenozoospermia. J. Endocrinol. Invest. 41, 781-787 (2017).

36. Zuccarello, D. et al. Mutations in dynein genes in patients affected by isolated non-syndromic asthenozoospermia. Hum. Reprod. 23, 1957-1962 (2008).

37. Kumar, P., Henikoff, S. \& Ng, P. C. Predicting the effects of coding nonsynonymous variants on protein function using the SIFT algorithm. Nat. Protoc. 4, 1073-1081 (2009).
38. Adzhubei, I. A. et al. A method and server for predicting damaging missense mutations. Nat. Methods 7, 248-249 (2010).

39. Jagadeesh, K. A. et al. M-CAP eliminates a majority of variants of uncertain significance in clinical exomes at high sensitivity. Nat. Genet. 48, 1581-1586 (2016).

40. Biasini, M. et al. SWISS-MODEL: modelling protein tertiary and quaternary structure using evolutionary information. Nucleic Acids Res. 42, 252-258 (2014).

41. DeLano, W. L. The PyMOL Molecular Graphics System. (DeLano Scientific, Palo Alto, CA, 2002).

42. Diaz, M. \& Pecinka, A. Scaffolding for repair: understanding molecular functions of the SMC5/6 complex. Genes 9, 36 (2018).

43. Wilhelm, L. et al. SMC condensin entraps chromosomal DNA by an ATP hydrolysis dependent loading mechanism in Bacillus subtilis. Elife. 4, e06659 (2015).

44. van Ruiten, M. S. \& Rowland, B. D. SMC complexes: universal DNA looping machines with distinct regulators. Trends Genet. 18, 30056-30058 (2018).

45. Andersen, J. S. et al. Proteomic characterization of the human centrosome by protein correlation profiling. Nature 426, 570 (2003).

46. Avidor-Reiss, T. et al. Decoding cilia function: defining specialized genes required for compartmentalized cilia biogenesis. Cell 117, 527-539 (2004).

47. Li, J. B. et al. Comparative genomics identifies a flagellar and basal body proteome that includes the BBS5 human disease gene. Cell 117, 541-552 (2004).

48. Chang, B. et al. In-frame deletion in a novel centrosomal/ciliary protein CEP290/NPHP6 perturbs its interaction with RPGR and results in early-onset retinal degeneration in the rd16 mouse. Hum. Mol. Genet. 15, 1847-1857 (2006).

49. De Rubeis, S. et al. Synaptic, transcriptional and chromatin genes disrupted in autism. Nature 515, 209 (2014).

50. Ververi, A., Splitt, M., Dean, J. C. S., DDD Study. \& Brady, A. F. Phenotypic spectrum associated with de novo mutations in QRICH1 gene. Clin. Genet. 93, 286-292 (2018).

51. Miki, K. et al. Targeted disruption of the Akap4 gene causes defects in sperm flagellum and motility. Dev. Biol. 248, 331-342 (2002).

52. Brown, P. R., Miki, K., Harper, D. B. \& Eddy, E. M. A-kinase anchoring protein 4 binding proteins in the fibrous sheath of the sperm flagellum. Biol. Reprod. 68, 2241-2248 (2003).

53. Matsuoka, Y. et al. Isolation and characterization of the spermatid-specific Smrp1 gene encoding a novel manchette protein. Mol. Reprod. Dev. 75, 967-975 (2008).

54. Iyengar, P. V., Hirota, T., Hirose, S. \& Nakamura, N. Membrane-associated RING-CH 10 (MARCH10 protein) is a microtubule-associated E3 ubiquitin ligase of the spermatid flagella. J. Biol. Chem. 286, 39082-39090 (2011).

55. Coutton, C., Arnoult, C. \& Ray, P. F. Commentary on "morphological characteristics and initial genetic study of multiple morphological anomalies of the flagella in China". Asian J. Androl. 18, 812 (2016).

56. Livak, K. J. \& Schmittgen, T. D. Analysis of relative gene expression data using real-time quantitative PCR and the 2(-Delta Delta C(T)) method. Methods $\mathbf{2 5}$, 402-408 (2001).

57. Doucette, L. P., Footz, T. \& Walter, M. A. FOXC1 regulates expression of prostaglandin receptors leading to an attenuated response to latanoprost. Invest. Ophthalmol. Vis. Sci. 6, 2548-2554 (2018).

58. Zhang, Q., Lei, X. \& Lu, H. Alterations of epigenetic signatures in hepatocyte nuclear factor $4 \alpha$ deficient mouse liver determined by improved ChIP-qPCR and (h)MeDIP-qPCR assays. PLoS ONE 1, e84925 (2014).

\section{Acknowledgements}

We are grateful to Prof. Huayu Qi (Guangzhou Institutes of Biomedicine and Health, Chinese Academy of Sciences) for providing the mouse monoclonal antibody against AKAP3 and Prof. Long Yu (Fudan University) for providing the rabbit polyclonal antibody against TSSK4. We thank all the patients who provided samples to this research. This work was supported by the National Nature Science fund of China (81370747, 31701085, and 31625015). This work was supported by the National Key Research and Development Program of China (SQ2018YFC1003603) and the National Nature Science fund of China $(81370747,31701085$, and 31625015).

\section{Author contributions}

Y.S. and H.Y. designed experiments, collected data, and wrote the manuscript. X.Y., F.L, X.J., W.L., and Y.Y. collected the blood, semen and testicular tissue samples and the volunteers' clinical information. X.Z., X.L., J.C., K.W., K.S., and M.L. performed the animal experiments. G.Y., X.P., Y.S., and S.L. carried out the electron microscopy experiments. K.C., W.T., and F.H. contributed to the CASA analyses. Y.S., C.J., J.X., and X.L. contributed to dual-luciferase reporter assays and CHIP-PCR. Y.S., J.S., H.W., and X.W. performed the PCR-sequencing analyses. L.Y., L.T., and W.K. carried out the Flow Cytometric Analysis and Cell Sorting assay. F.Z., Y.S., and W.X. supervised the study and wrote the manuscript. 


\section{Additional information}

Supplementary Information accompanies this paper at https://doi.org/10.1038/s41467018-08182-x.

Competing interests: The authors declare no competing interests.

Reprints and permission information is available online at http://npg.nature.com/ reprintsandpermissions/

Journal peer review information: Nature Communications thanks the anonymous reviewers for their contribution to the peer review of this work.

Publisher's note: Springer Nature remains neutral with regard to jurisdictional claims in published maps and institutional affiliations. (c) (i) Open Access This article is licensed under a Creative Commons Attribution 4.0 International License, which permits use, sharing, adaptation, distribution and reproduction in any medium or format, as long as you give appropriate credit to the original author(s) and the source, provide a link to the Creative Commons license, and indicate if changes were made. The images or other third party material in this article are included in the article's Creative Commons license, unless indicated otherwise in a credit line to the material. If material is not included in the article's Creative Commons license and your intended use is not permitted by statutory regulation or exceeds the permitted use, you will need to obtain permission directly from the copyright holder. To view a copy of this license, visit http://creativecommons.org/ licenses/by/4.0/.

(C) The Author(s) 2019 